\title{
Dynamics and Stability of Low-Reynolds-Number Swimming Near a Wall*
}

\author{
Yizhar Or $^{\dagger}$, Sebastian Zhang ${ }^{\ddagger}$, and Richard M. Murray ${ }^{\S}$
}

\begin{abstract}
The locomotion of microorganisms and tiny artificial swimmers is governed by low-Reynolds-number hydrodynamics, where viscous effects dominate and inertial effects are negligible. While the theory of low-Reynolds-number locomotion is well studied for unbounded fluid domains, the presence of a boundary has a significant influence on the swimmer's trajectories and poses problems of dynamic stability of its motion. In this paper we consider a simple theoretical model of a microswimmer near a wall, study its dynamics, and analyze the stability of its motion. We highlight the underlying geometric structure of the dynamics, and establish a relation between the reversing symmetry of the system and existence and stability of periodic and steady solutions of motion near the wall. The results are demonstrated by numerical simulations and validated by motion experiments with macroscale robotic swimmer prototypes.
\end{abstract}

Key words. dynamics of locomotion, low-Reynolds swimming, dynamic stability

AMS subject classifications. 37J15, 37J25, 76M60

DOI. $10.1137 / 100808745$

1. Introduction. The locomotion of microorganisms, as well as of futuristic miniature robotic swimmers for biomedical applications, is governed by low-Reynolds-number hydrodynamics [30, 40,60]. Reynolds number, which encompasses the ratio of inertial forces to viscous forces, is defined as $R e=V L / \nu$, where $V$ is a characteristic velocity, $L$ is a characteristic length scale, and $\nu$ is the kinematic viscosity of the fluid. For example, a typical Reynolds number for a human swimmer who is governed by inertial effects is on the order of $10^{4}$, whereas microorganisms typically have $R e \approx 10^{-4}$ and swim by harnessing viscous effects. The theory of low-Re locomotion of microorganisms and motile cells in nature has been widely studied in the physics, fluid mechanics, and biology literature, e.g., [12, 46, 58]. In the context of nanotechnology and engineering, some efforts to develop miniaturized swimmers, primarily for biomedical applications, were reported in [4, 21, 26, 42, 70]. While a vast majority of the theoretical works use the simplifying assumption of unbounded fluid domain, in realistic scenarios microswimmers often move in confined environments and interact with the boundaries. The presence of solid boundaries has profound effects on the dynamics and motion trajectories of low-Re swimmers, as confirmed by numerical simulations $[23,28,61,72]$ and observed in several laboratory experiments with swimming microorganisms [14, 25, 45].

\footnotetext{
${ }^{*}$ Received by the editors September 15, 2010; accepted for publication (in revised form) by T. Kaper April 23, 2011; published electronically September 20, 2011. Preliminary versions of these results appeared previously in [55] and [73].

http://www.siam.org/journals/siads/10-3/80874.html

${ }^{\dagger}$ Faculty of Mechanical Engineering, Technion - Israel Institute of Technology, Haifa 32000, Israel (izi@tx.technion. ac.il).

¥Department of Mechanical Engineering, Clemson University, Clemson, SC 29634 (szhang1024@gmail.com).

${ }^{\S}$ Department of Control and Dynamical Systems, California Institute of Technology, Pasadena, CA 91125 (murray@cds.caltech.edu).
}

1013

Copyright (C) by SIAM. Unauthorized reproduction of this article is prohibited. 


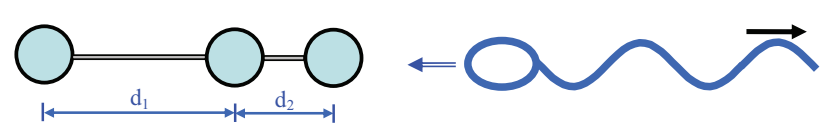

(a) (b)

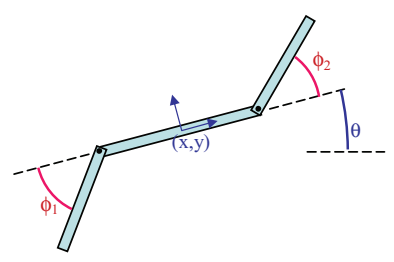

(c)

Figure 1. Simplified low-Re swimmer models: (a) the three-linked spheres [53], (b) a eukaryote such as a sperm cell waving a flagellar tail, (c) Purcell's three-link swimmer.

The goal of this work is to investigate these effects from the viewpoint of dynamical systems and control theory, by studying a simplified theoretical model of a low-Re swimmer near an infinite plane wall. In this study, which highlights the geometric structure of the swimmer's dynamic equations, we focus on stability of the motion near a wall.

In the limiting case of $R e \rightarrow 0$, the motion of the fluid is governed by Stokes equations of viscous flow, and the motion of the swimmer is assumed to be quasi-static; that is, it maintains static equilibrium at all times. A typical strategy for low-Re swimming is changing the internal configuration of the swimmer in a nonreciprocal way in order to generate net motion. Due to the complex interaction between the motion of the fluid and the body, this problem has been studied theoretically under several simplifications. One approach focuses on axisymmetric swimmers, whose motion is only one-dimensional along the axis of symmetry $[1,47,53]$. As an example, Figure 1(a) shows the three-linked-spheres swimmer studied in [53], which is propelled along its axis by changing the separation distances, $d_{1}$ and $d_{2}$, between the spheres periodically in an inchworm-like fashion. Another approach studies biological mechanisms of ciliary and flagellar propulsion in microorganisms by modeling them as a small-amplitude traveling waves $[6,7,49,69]$, as illustrated in Figure 1(b). The one-dimensional motion of the swimmer is then formulated by using perturbation methods. In more general models, the swimmer translates and rotates in two or three dimensions under cyclic shape changes of finite amplitude [71]. A classical example is Purcell's articulated three-link swimmer model [3, 60], which generates planar translation and rotation by changing its interlink joint angles $\phi_{1}$ and $\phi_{2}$; see Figure 1(c). Denoting the coordinates describing the configuration of the swimmer as $\mathbf{q}$ and regarding the rate of shape changes as controlled inputs $\mathbf{u}(t)$, the motion of the swimmer is governed by a driftless nonlinear control system $[10,13]$ of the form $\dot{\mathbf{q}}=\mathbf{G}(\mathbf{q}) \mathbf{u}$. In case where the fluid domain is assumed to be unbounded, the equation of motion enjoys an additional geometric structure called gauge symmetry [2, 41, 65], for which the equation of motion is invariant under rigid-body transformation of the swimmer's body. This property enables formulation of the geometric phase, which is the rigid-body translation and rotation associated with a given cyclic change of internal shape. This concept has been widely explored in the context of geometric mechanics and locomotion of robotic systems [39, 56, 64] and also for control of swimming in inviscid fluid (i.e., the case of $R e \rightarrow \infty$ ) [35, 51, 52]. Another particular subclass of swimmers with simplified dynamics is treadmilling swimmers, which propel themselves by generating purely tangential motion along their boundary while their shape remains fixed [32, 47, 48]. For those swimmers, the equation of motion is simplified further, as it does not depend on the swimmer's unchanging shape.

Copyright (c) by SIAM. Unauthorized reproduction of this article is prohibited. 
A key limitation of the theoretical models described above is that they consider unbounded fluid domain only, whereas in reality, microswimmers often swim in confined environments and interact with the boundary. The leading-order effect of an infinite plane wall on the motion of inert rigid bodies in Stokes flow was analyzed in [8, 9, 37]. The motion of microorganisms with flagellar propulsion near a plane wall was analyzed in [36] under the simplified model of an infinite waving sheet, where the swimmer's net motion is assumed to remain one-dimensional and parallel to the wall. When finite-length swimmers are considered, the presence of the wall can significantly change their motion trajectories into two- and even three-dimensional curves. Biological examples are E. coli swimming in circles above a flat surface [45], accumulation of bacteria and sperm cells near boundaries [5, 14], shear-induced periodic orbits of bacteria and larvae [38, 74], and interesting "dancing" motion of pairs of Volvox algae under the combined effects of gravity and a near wall [20]. Some of the works mentioned above used simple theoretical models to provide physical explanations for these wall effects, and some of the phenomena were observed in numerical simulations or experiments. In particular, the work of Berke et al. [5] models a low-Re swimmer near a wall as a moving force-dipole singularity in order to explain the accumulation of swimming bacteria near a solid surface. Under this simplifying model, which considers far-field effects of the wall, closed-form expressions are derived for the attraction of the swimmer towards the wall and for its rotational reorientation parallel to the wall. However, this model is singular in the sense that it predicts that the swimmer crashes into the wall in finite time, in contrast to experimental observations of settling to a small but finite distance from the wall. Other recent works study the attraction of flagellated bacteria and sperm cells to solid boundaries by utilizing efficient numerical techniques for long-time dynamic simulations [66, 67]. The simulation results clearly show attraction of the swimmers towards the wall, followed by settling to a nonzero separation distance and a slightly inclined orientation. These works also numerically investigate the influence of changing relative sizes of the swimmer's head and tail on the convergence of motion, though they lack simple physical explanations and insights.

The goal of this paper is to bridge the gap between the aforementioned works [5] and $[66,67]$ and to consider a simple theoretical model of a low-Re swimmer which will enable gaining insights into the dynamics of low-Re swimming near a wall. The chosen model consists of an assemblage of spheres mounted to a thin rigid structure, which are actuated by rotation about their attachment point. The advantage of this model is that the shape of the swimmer remains fixed, which enables focusing solely on the dynamic interaction of the swimmer and the boundary without the complication associated with the influence of shape changes. Moreover, the choice of spheres as the basic building blocks enables using known approximate expressions for the sphere-sphere and sphere-wall hydrodynamic interactions [22, 40, 68]. We highlight the geometric structure of the swimmer's dynamics in analysis of the motion near the wall. In particular, we make use of the reversing symmetry [43, 44, 63] of the swimmer-wall configuration and its implications on the dynamic stability of steady translation as well as periodic oscillations along the wall. Note that existence of periodic motion in viscous shear flow was already studied in works such as [33] for an inert rigid body and in [74] for self-propelled larvae. The works $[11,34]$ study periodic motions of interacting rigid bodies sedimenting in unbounded viscous fluid, and the work of Golubitsky, Krupa, and Lim [27] formally establishes the relation between existence of periodic solutions and the reversing symmetry of the

Copyright (c) by SIAM. Unauthorized reproduction of this article is prohibited. 
system. Nevertheless, a key contribution of our work is showing that the presence of a plane wall also induces a reversing symmetry, which, in turn, implies existence of periodic solutions of oscillations along the wall which are neutrally stable. Next, we analyze the case where the structure of the swimmer breaks the reversing symmetry. We then show that the solutions of steady translation and of periodic oscillations along the wall can be made open-loop asymptotically stable. The implications of this key result are twofold. First, in the context of biological microswimmers, it provides additional insight into the hydrodynamical mechanism of accumulation near surfaces with a clear dynamical interpretation. Second, in the context of artificial swimmers for biomedical applications, this result implies that they can be steered in confined environments by using open-loop commands only, without requiring on-board sensing and control for stabilization. This fact can potentially be utilized as a guideline towards minimalistic design of such swimmers in the future. Finally, we qualitatively corroborate the theoretical predictions by presenting results of motion experiments of macroscale robotic prototypes swimming near a wall in a viscous fluid.

The organization of the paper is as follows. In section 2 , the governing hydrodynamic equations are formulated, resulting in a driftless nonlinear control system describing the swimmer's motion near a wall. Section 3 analyzes the dynamics of the simplest swimmer model, which is composed of two rotating spheres. This simple swimmer possesses a reversing symmetry which implies the co-existence of neutrally stable solutions of steady translation and periodic oscillations along the wall. Section 4 considers the $2+1$-sphere swimmer model, in which the reversing symmetry is broken, and characterizes the dynamic stability of its motion near the wall. Section 5 reports the experimental results. Finally, the concluding section discusses limitations of the results and lists possible directions for future extension. A brief version of the theoretical analysis in this paper has been published in the short paper [55], and this paper complements it by detailed derivation of the governing equations thorough numerical investigation and discussion and by verification by experimental results. The experimental results have also been reported in the conference paper [73].

2. Problem formulation. This section formulates the dynamics of a microswimmer model composed of a rigid structure of spheres which are actuated by rotation about their point of attachment to the structure. First, the low-Re fluid-mechanical governing equations are formulated, followed by derivation of the approximate mobility matrices, which relate hydrodynamic forces to velocities. Then the dynamic equations of planar motion of the swimmer near a wall are formulated as a driftless nonlinear control system.

2.1. Hydrodynamic model. Consider a collection of $n$ neutrally buoyant rigid spherical particles submerged in a quiescent fluid which is viscous and incompressible. The fluid domain is bounded by an infinite plane wall, denoted $\mathcal{W}$. The position of the center of the $i$ th particle is denoted $\mathbf{x}_{i}$, and its linear and angular velocities are denoted by $\mathbf{U}_{i}$ and $\boldsymbol{\Omega}_{i}$, respectively. Assuming that the Reynolds number is vanishingly small, the motion of the fluid is governed by Stokes equations, which are given by

$$
-\nabla p+\mu \nabla^{2} \mathbf{v}=0, \quad \nabla \cdot \mathbf{v}=0,
$$

where $\mathbf{v}$ is the fluid velocity field, $p$ is the pressure distribution, and $\mu$ is the fluid viscosity. 
The boundary conditions for the velocity field are given by

$$
\mathbf{v}(\mathbf{x})= \begin{cases}0, & \mathbf{x} \in \mathcal{W}, \\ 0, & \|\mathbf{x}\| \rightarrow \infty, \\ \mathbf{U}_{i}+\boldsymbol{\Omega}_{i} \times\left(\mathbf{x}-\mathbf{x}_{i}\right), & \mathbf{x} \in S_{i}, \quad i=1, \ldots, n\end{cases}
$$

where $S_{i}$ denotes the boundary of the $i$ th particle. Physically, conditions (2) impose zero relative slip of the fluid on the boundaries of the moving particles and on the stationary wall, and they also require that the fluid velocity vanish at infinity. For given instantaneous velocities of the particles $\mathbf{U}_{i}, \boldsymbol{\Omega}_{i}$, the fluid velocity $\mathbf{v}(\mathbf{x})$ and pressure $p(\mathbf{x})$ are determined according to (1) and (2). However, the motions of the particles and the fluid are coupled through the forces and torques acting between them, which are formulated as follows. Let $\mathbf{F}_{i}$ denote the net force exerted by the fluid on the $i$ th particle, and let $\mathbf{L}_{i}$ denote the net torque exerted by the fluid about the center of the $i$ th particle. These net force and torque are obtained by integrating the fluid stress over the entire surface of the particle $S_{i}$, and are given by

$$
\begin{aligned}
\mathbf{F}_{i} & =\iint_{S_{i}} \boldsymbol{\sigma} \cdot \mathbf{n} d s \\
\mathbf{L}_{i} & =\iint_{S_{i}}\left(\mathbf{x}-\mathbf{x}_{i}\right) \times(\boldsymbol{\sigma} \cdot \mathbf{n}) d s,
\end{aligned}
$$

where $\mathbf{n}$ is a unit normal to the boundary $S_{i}$ at $\mathbf{x} ; d s$ is an area element; $\boldsymbol{\sigma}$ is the local stress tensor of the fluid, given by $\boldsymbol{\sigma}=-p \mathbf{I}+\mu\left(\nabla \mathbf{v}+\nabla^{T} \mathbf{v}\right)$; and $\mathbf{I}$ is the identity matrix. Despite the complex coupling between (1), (2), and (3), a fundamental property that stems from the linearity of Stokes equations is the existence of a linear relation between forces/torques and velocities of the particles, as follows. Let $\mathbf{U}=\left(\mathbf{U}_{1} \ldots \mathbf{U}_{n} \boldsymbol{\Omega}_{1} \ldots \boldsymbol{\Omega}_{n}\right)^{T}$ be a vector augmenting the linear and angular velocities of all particles. Similarly, let $\mathbf{F}=\left(\mathbf{F}_{1} \ldots \mathbf{F}_{n} \mathbf{L}_{1} \ldots \mathbf{L}_{n}\right)^{T}$ be the vector augmenting the net forces and torques exerted by the fluid on the particles. Then the linear relation between velocities and forces is expressed as (cf. [30])

$$
\mathbf{F}=\mathbf{R U}
$$

The matrix $\mathbf{R}$ is called the resistance matrix, and depends only on the positions of all particles. A fundamental property of $\mathbf{R}$ is that it is symmetric and positive definite [30]. Additionally, it is not diagonal or block-diagonal, a fact that represents the hydrodynamic coupling between the particles. Nevertheless, the magnitude of the coupling terms between two particles is monotonically decreasing as a function of the separation distance between them.

In many cases, it is more convenient to work with the inverse relation $\mathbf{U}=\mathbf{M F}$, where $\mathbf{M}=\mathbf{R}^{-1}$ is called the mobility matrix. The mobility matrix can be decomposed into blocks in the following way, demonstrated for two particles with indices $\alpha$ and $\beta$ :

$$
\left(\begin{array}{c}
\mathbf{U}_{\alpha} \\
\mathbf{U}_{\beta} \\
\boldsymbol{\Omega}_{\alpha} \\
\boldsymbol{\Omega}_{\beta}
\end{array}\right)=\left(\begin{array}{llll}
\mathbf{M}_{U F}^{\alpha \alpha} & \mathbf{M}_{U F}^{\alpha \beta} & \mathbf{M}_{U L}^{\alpha \alpha} & \mathbf{M}_{U L}^{\alpha \beta} \\
\mathbf{M}_{U F}^{\beta \alpha} & \mathbf{M}_{U F}^{\beta \beta} & \mathbf{M}_{U L}^{\beta \alpha} & \mathbf{M}_{U L}^{\beta \beta} \\
\mathbf{M}_{\Omega F}^{\alpha \alpha} & \mathbf{M}_{\Omega F}^{\alpha \beta} & \mathbf{M}_{\Omega L}^{\alpha \alpha} & \mathbf{M}_{\Omega L}^{\alpha \beta} \\
\mathbf{M}_{\Omega F}^{\beta \alpha} & \mathbf{M}_{\Omega F}^{\beta \beta} & \mathbf{M}_{\Omega L}^{\beta \alpha} & \mathbf{M}_{\Omega L}^{\beta \beta}
\end{array}\right)\left(\begin{array}{c}
\mathbf{F}_{\alpha} \\
\mathbf{F}_{\beta} \\
\mathbf{L}_{\beta} \\
\mathbf{L}_{\beta}
\end{array}\right) .
$$

Copyright $\odot$ by SIAM. Unauthorized reproduction of this article is prohibited. 
Since the mobility matrix is also symmetric, its matrix blocks in (5) are related as $\mathbf{M}_{U F}^{\alpha \beta}=$ $\left(\mathbf{M}_{U F}^{\beta \alpha}\right)^{T}, \mathbf{M}_{\Omega F}^{\alpha \beta}=\left(\mathbf{M}_{U L}^{\beta \alpha}\right)^{T}$, and so on. For more than two particles, the mobility matrix is constructed similarly, where each block represents the hydrodynamic interaction of a pair of particles or a particle with itself. In general, the exact dependence of the resistance or mobility matrices on the configuration cannot be formulated explicitly, except for special cases such as a single spheroidal particle or two spheres [30]. However, in many cases, $\mathbf{R}$ or $\mathbf{M}$ can be approximated under certain scaling arguments and physical assumptions. In this work, we adopt the hydrodynamic model proposed by Swan and Brady [68] for far-field approximation of the mobility matrix, as detailed next.

2.2. Far-field approximation of the mobility matrix. We now review the derivation of the far-field approximation of the mobility matrix in [68], for completeness of the presentation. Readers who are less interested in the details of the fluid-mechanical model may skip to subsection 2.3. The model in [68] considers multiple spherical particles in the presence of a plane wall, assuming that the sphere-sphere and sphere-wall separation distances are large compared to the radii of the spheres. For convenience, we assume that all spheres have equal radius $a$. The basic building block in this model is the Green's function, which gives the singular solution for the fluid velocity field $\mathbf{v}(\mathbf{x})$ in (1) under a concentrated force applied at a point $\mathbf{y}$. Green's function for unbounded fluid (also known as Stokeslet) is given by

$$
\mathbf{G}_{0}(\mathbf{x} ; \mathbf{y})=\frac{1}{8 \pi \mu}\left(\frac{\mathbf{I}}{\|\mathbf{x}-\mathbf{y}\|}+\frac{(\mathbf{x}-\mathbf{y})(\mathbf{x}-\mathbf{y})}{\|\mathbf{x}-\mathbf{y}\|^{3}}\right)
$$

For a concentrated force $\mathbf{f}$ applied at a point $\mathbf{y}$, the fluid velocity is obtained as $\mathbf{v}(\mathbf{x})=$ $\mathbf{G}_{0}(\mathbf{x} ; \mathbf{y}) \mathbf{f}$. In the case where the fluid is bounded by an infinite plane wall, the Green's function is modified in order to account for the no-slip boundary condition on the wall, and is given by [68]:

$$
\mathbf{G}(\mathbf{x}, \mathbf{y})=\mathbf{G}_{0}(\mathbf{x}, \mathbf{y})-\mathbf{G}_{0}\left(\mathbf{x}, \mathbf{y}^{\prime}\right)+h(\mathbf{y})^{2} \nabla_{\mathbf{y}^{\prime}}^{2} G_{0}\left(\mathbf{x}, \mathbf{y}^{\prime}\right) \cdot \mathbf{P}+2 h(\mathbf{y})\left(\mathbf{P} \cdot \nabla_{\mathbf{y}^{\prime}}^{2} \mathbf{G}_{0}\left(\mathbf{x}, \mathbf{y}^{\prime}\right) \cdot \mathbf{n}_{w}\right)^{T}
$$

where $\mathbf{w}$ is a point on the wall, $\mathbf{n}_{w}$ is an outward unit normal to the wall, $h(\mathbf{y})=\mathbf{n}_{w} \cdot \mathbf{y}, \mathbf{P}=$ $\mathbf{I}-2 \mathbf{n}_{w} \mathbf{n}_{w}^{T}$, and $\mathbf{y}^{\prime}=\mathbf{P} \cdot \mathbf{y}$ is the "image point" of $\mathbf{y}$ beyond the wall. Exploiting the linearity of Stokes equations (1), the Green's function can be utilized to obtain the fluid velocity induced by a force distribution $\mathbf{f}(\mathbf{y})$ along the boundary $S_{\beta}$ of a sphere $\beta$ via integration as

$$
\mathbf{v}^{\prime}(\mathbf{x})=\iint_{S_{\beta}} \mathbf{G}(\mathbf{x}, \mathbf{y}) \cdot \mathbf{f}(\mathbf{y}) d s
$$

The next step is to expand (8) in surface moments of the force distribution $\mathbf{f}(\mathbf{y})$ to obtain

$$
\mathbf{v}^{\prime}(\mathbf{x})=\left.\left(1+\frac{a^{2}}{6} \nabla_{\mathbf{y}}^{2}\right) \mathbf{G}(\mathbf{x}, \mathbf{y})\right|_{\mathbf{y}=\mathbf{x}_{\beta}} \cdot \mathbf{F}_{\beta}+\frac{1}{2} \nabla_{\mathbf{y}}^{2} \times\left.\mathbf{G}(\mathbf{x}, \mathbf{y})\right|_{\mathbf{y}=\mathbf{x}_{\beta}} \cdot \mathbf{L}_{\beta}+\cdots
$$


Note that we truncate the expansion in the moment level, while [22] and [68] also include the next term of stresslet (i.e., in the terminology of [22], we choose to work with the F-T version). The final ingredient is Faxén's formula, which relates the disturbance velocity field $\mathbf{v}^{\prime}(\mathbf{x})$ around a sphere $\alpha$ to its linear and angular velocities, as follows:

$$
\begin{aligned}
& \mathbf{U}_{\alpha}=\frac{\mathbf{F}_{\alpha}}{6 \pi \mu a}+\left.\left(1+\frac{a^{2}}{6} \nabla_{\mathbf{x}}^{2}\right) \mathbf{v}^{\prime}(\mathbf{x})\right|_{\mathbf{x}=\mathbf{x}_{\alpha}}, \\
& \boldsymbol{\Omega}_{\alpha}=\frac{\mathbf{L}_{\alpha}}{8 \pi \mu a^{3}}+\frac{1}{2} \nabla_{\mathbf{x}} \times\left.\mathbf{v}^{\prime}(\mathbf{x})\right|_{\mathbf{x}=\mathbf{x}_{\alpha}} .
\end{aligned}
$$

Combining (9) and (10), one obtains the hydrodynamic interaction between two particles $\alpha$ and $\beta$, which is given by the block mobility matrices of (5) as

$$
\begin{aligned}
& \mathbf{M}_{U F}^{\alpha \beta}=\left.\left(1+\frac{a^{2}}{6} \nabla_{\mathbf{x}}^{2}\right)\left(1+\frac{a^{2}}{6} \nabla_{\mathbf{y}}^{2}\right) \mathbf{G}(\mathbf{x}, \mathbf{y})\right|_{\mathbf{x}=\mathbf{x}_{\alpha}} ^{\mathbf{y}=\mathbf{x}_{\beta}}, \\
& \mathbf{M}_{\Omega F}^{\alpha \beta}=\frac{1}{2} \nabla_{\mathbf{x}}^{2} \times\left.\left(1+\frac{a^{2}}{6} \nabla_{\mathbf{y}}^{2}\right) \mathbf{G}(\mathbf{x}, \mathbf{y})\right|_{\mathbf{x}=\mathbf{x}_{\alpha}} ^{\mathbf{y}=\mathbf{x}_{\beta}}, \\
& \mathbf{M}_{\Omega L}^{\alpha \beta}=\frac{1}{2} \nabla_{\mathbf{x}}^{2} \times \frac{1}{2} \nabla_{\mathbf{y}}^{2} \times\left.\mathbf{G}(\mathbf{x}, \mathbf{y})\right|_{\mathbf{x}=\mathbf{x}_{\alpha}} ^{\mathbf{y}=\mathbf{x}_{\beta}} .
\end{aligned}
$$

Note that when accounting for the self-interaction terms of the particle $\alpha$ with itself (i.e., when $\alpha=\beta$ ), the disturbance velocity $\mathbf{v}^{\prime}(\mathbf{x})$ in Faxén's formula (10) is computed by replacing the Green's function $\mathbf{G}(\mathbf{x}, \mathbf{y})$ in (6) with $\mathbf{G}_{w}(\mathbf{x}, \mathbf{y})=\mathbf{G}(\mathbf{x}, \mathbf{y})-\mathbf{G}_{0}(\mathbf{x}, \mathbf{y})$. That is, only the reflection term is counted, while the singular Stokeslet term is discarded. Thus, the expressions of the "self" mobility block matrices are given by

$$
\begin{aligned}
& \mathbf{M}_{U F}^{\alpha \alpha}=\frac{\mathbf{I}}{6 \pi \mu a}+\left.\left(1+\frac{a^{2}}{6} \nabla_{\mathbf{x}}^{2}\right)\left(1+\frac{a^{2}}{6} \nabla_{\mathbf{y}}^{2}\right) \mathbf{G}_{w}(\mathbf{x}, \mathbf{y})\right|_{\mathbf{x}=\mathbf{x}_{\alpha}} ^{\mathbf{y}=\mathbf{x}_{\alpha}}, \\
& \mathbf{M}_{\Omega F}^{\alpha \alpha}=\frac{1}{2} \nabla_{\mathbf{x}}^{2} \times\left.\left(1+\frac{a^{2}}{6} \nabla_{\mathbf{y}}^{2}\right) \mathbf{G}_{w}(\mathbf{x}, \mathbf{y})\right|_{\mathbf{x}=\mathbf{x}_{\alpha}} ^{\mathbf{y =}=\mathbf{x}_{\alpha}}, \\
& \mathbf{M}_{\Omega L}^{\alpha \alpha}=\frac{\mathbf{I}}{8 \pi \mu a^{3}}+\frac{1}{2} \nabla_{\mathbf{x}}^{2} \times \frac{1}{2} \nabla_{\mathbf{y}}^{2} \times\left.\mathbf{G}_{w}(\mathbf{x}, \mathbf{y})\right|_{\mathbf{x}=\mathbf{x}_{\alpha}} ^{\mathbf{y}=\mathbf{x}_{\alpha}} .
\end{aligned}
$$

The resulting explicit expressions for the block mobility matrices, detailed in [68], are highly complicated. As a simple example, we give here the mobility matrix for two spheres of equal radii $a=1$ whose center-center line is parallel to the $y$-axis near a plane wall at $y=0$, as shown in Figure 2(a). Due to symmetry of the configuration, we focus here on motion in the $x y$-plane only. The mobility relation of velocities in the $x y$-plane and angular velocities 


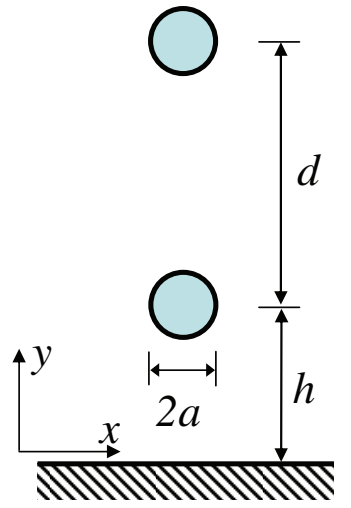

(a)

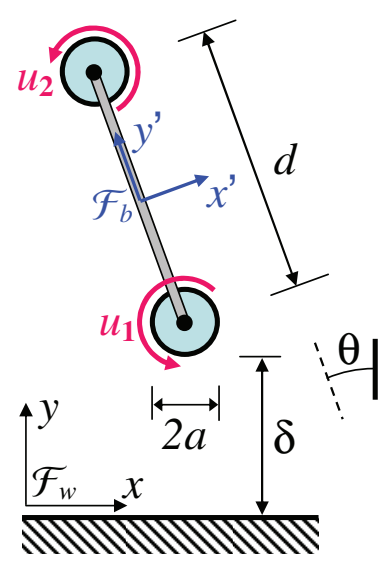

(b)

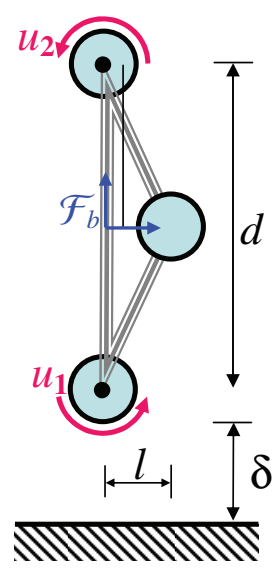

(c)

Figure 2. (a) Two spheres near a wall. (b) The two-sphere swimmer model. (c) The 2+1-sphere swimmer model.

about the $z$-axis with linear forces in the $x y$-plane and torques about the $z$-axis is given by

$$
\left(\begin{array}{l}
\mathbf{U}_{1 x} \\
\mathbf{U}_{1 y} \\
\mathbf{U}_{2 x} \\
\mathbf{U}_{2 y} \\
\boldsymbol{\Omega}_{1 z} \\
\boldsymbol{\Omega}_{2 z}
\end{array}\right)=-\frac{1}{6 \pi \mu}\left(\begin{array}{cccccc}
m_{11} & 0 & m_{13} & 0 & m_{15} & m_{16} \\
0 & m_{22} & 0 & m_{24} & 0 & 0 \\
m_{13} & 0 & m_{33} & 0 & m_{35} & m_{36} \\
0 & m_{24} & 0 & m_{44} & 0 & 0 \\
m_{15} & 0 & m_{35} & 0 & m_{55} & m_{56} \\
m_{16} & 0 & m_{36} & 0 & m_{56} & m_{66}
\end{array}\right)\left(\begin{array}{l}
\mathbf{F}_{1 x} \\
\mathbf{F}_{1 y} \\
\mathbf{F}_{2 x} \\
\mathbf{F}_{2 y} \\
\mathbf{L}_{1 z} \\
\mathbf{L}_{2 z}
\end{array}\right),
$$

where

$$
\begin{aligned}
& m_{11}=1-\frac{9}{16 h}+\frac{1}{8 h^{3}}-\frac{1}{16 h^{5}}, \quad m_{22}=1-\frac{9}{8 h}+\frac{1}{2 h^{3}}-\frac{1}{8 h^{5}}, \\
& m_{13}=\frac{3}{4 d}+\frac{1}{2 d^{3}}-\frac{3}{4(d+2 h)}+\frac{1}{(d+2 h)^{3}}-\frac{2}{(d+2 h)^{5}}-\frac{3(d+h)}{2(d+2 h)^{2}}+\frac{3(d+h)^{2}}{2(d+2 h)^{3}}, \\
& m_{15}=-\frac{3}{32 h^{4}}, \quad m_{16}=\frac{3}{4 d^{2}}-\frac{3}{4(d+2 h)^{2}}+\frac{3 h}{2(d+2 h)^{3}}-\frac{3}{2(d+2 h)^{4}}, \\
& m_{24}=\frac{3}{2 d}-\frac{1}{d^{3}}-\frac{3}{2(d+2 h)}+\frac{4}{(d+2 h)^{3}}-\frac{4}{(d+2 h)^{5}}-\frac{3(d+h)}{(d+2 h)^{2}}+\frac{3(d+h)^{2}}{(d+2 h)^{3}}, \\
& m_{33}=1-\frac{9}{16(d+h)}+\frac{1}{8(d+h)^{3}}-\frac{1}{16(d+h)^{5}}, \quad 3 \quad \frac{3(d+h)}{2(d+2 h)^{3}}, \\
& m_{35}=-\frac{3}{4 d^{2}}-\frac{3}{4(d+2 h)^{2}}-\frac{3}{2(d+2 h)^{4}}+\frac{1}{8(d+h)^{3}}-\frac{1}{8(d+h)^{5}}, \\
& m_{36}=-\frac{3}{32(d+h)^{4}}, \quad m_{44}=1-\frac{9}{8(d+h)}+\frac{1}{2(d+h} . \\
& m_{55}=\frac{3}{4}-\frac{15}{64 h^{3}}, \quad m_{56}=-\frac{3}{8 d^{3}}-\frac{15}{8(d+2 h)^{3}}, \quad m_{66}=\frac{3}{4}-\frac{15}{64(d+h)^{3}} .
\end{aligned}
$$


2.3. The dynamics of planar swimming. We now utilize the hydrodynamic relations derived above in order to formulate the dynamics of swimming as a control system. Consider a simple model of a microswimmer composed of $n$ rigid spheres of radius $a$ whose centers lie within the $x y$-plane. The spheres are connected by a rigid structure of thin rods, called the body of the swimmer. The swimmer is submerged in a quiescent viscous fluid which is bounded by an infinite plane wall at $y=0$ (Figure 2(b)-(c)). The spheres labeled $1, \ldots, m$ are actuated by rotation about their $z$-axis which is fixed to the body. Note that, even though the fluid motion in this case is three-dimensional, symmetry of the spheres' configuration about the plane $z=0$ implies that the motion of the swimmer is restricted to the $x y$-plane. Therefore, all vectors of position, linear velocity, and forces will be considered as elements of $\mathbb{R}^{2}$ representing their $(x, y)$-components, while torques and angular velocities will be considered as scalars representing the component around $z$-axis, as in (13). Let $\mathcal{F}_{w}$ be a world-fixed reference frame, and let $\mathcal{F}_{b}$ be a reference frame attached to the swimmer's body. Let $u_{i} \in \mathbb{R}$ be the angular velocity of the $i$ th sphere with respect to the body, which is assumed to be controlled by the swimmer, and denote $\mathbf{u}=\left(u_{1}, \ldots, u_{m}\right)^{T}$. Let $\mathbf{q}=(x, y, \theta)^{T} \in S E(2)$ denote the position and orientation of the body frame $\mathcal{F}_{b}$ in $x y$-plane, expressed in the world frame $\mathcal{F}_{w}$. Let $\mathbf{r}_{i} \in \mathbb{R}^{2}$ be the constant position vector of the $i$ th sphere expressed in the body frame $\mathcal{F}_{b}$. The absolute position of the $i$ th sphere is thus given by

$$
\mathbf{x}_{i}=\mathbf{r}_{b}+\mathbf{D}(\theta) \mathbf{r}_{i}, \quad \text { where } \quad \mathbf{r}_{b}=(x, y) \quad \text { and } \quad \mathbf{D}(\theta)=\left(\begin{array}{rr}
\cos \theta & -\sin \theta \\
\sin \theta & \cos \theta
\end{array}\right)
$$

The linear velocity of each sphere is obtained by differentiation of (14) as

$$
\dot{\mathbf{x}}_{i}=\mathbf{r}_{b}+\mathbf{J D}(\theta) \mathbf{r}_{i} \dot{\theta}, \quad \text { where } \mathbf{J}=\left(\begin{array}{rr}
0 & -1 \\
1 & 0
\end{array}\right) .
$$

The angular velocity of each sphere about the $z$-axis is given by

$$
\omega_{i}= \begin{cases}\dot{\theta}+u_{i}, & 1 \leq i \leq m, \\ \dot{\theta}, & i>m .\end{cases}
$$

Using only planar components, the vector of spheres' velocities is $\mathbf{U}=\left(\dot{\mathbf{x}}_{1}, \ldots, \dot{\mathbf{x}}_{1}, \omega_{1}, \ldots, \omega_{n}\right)^{T}$. The velocity relations in (15) and (16) can then be written in matrix form as

$$
\mathbf{U}=\mathbf{T} \dot{\mathbf{q}}+\mathbf{E u} .
$$

The key point in determining the instantaneous velocity of the swimmer is the fact that under the low-Reynolds-number assumption, the motion of the swimmer is quasi-static, implying that the net force and torque acting on it must vanish. Let $\mathbf{F}_{b}=\left(f_{x}, f_{y}, \tau_{z}\right)$ denote the net force and torque acting on the swimmer's rigid frame $\mathcal{F}_{b}$. It is assumed that the hydrodynamic resistance of the thin rigid frame is negligible compared to that of the spheres, so that the hydrodynamic forces and torques acting on each sphere are transferred directly to the body. The net force and torque on the body are thus given by $\mathbf{F}_{b}=\mathbf{F}_{1}+\cdots+\mathbf{F}_{n}$ and $\tau_{b}=\mathbf{r}_{1}^{T} \mathbf{J}^{T} \mathbf{F}_{1}+\mathbf{L}_{1 z}+\cdots+\mathbf{r}_{n}^{T} \mathbf{J}^{T} \mathbf{F}_{n}+\mathbf{L}_{n z}$. Using only the $(x, y)$-components of the forces $\mathbf{F}_{i}$ 
and the $z$-component of the torques $\mathbf{L}_{i}$ as in (13), it can be verified that in matrix form this relation is simply given by

$$
\mathbf{F}_{b}=\mathbf{T}^{T} \mathbf{F}
$$

Substituting the resistance relation (4) and the kinematic relations (17) and (18) into the quasi-static equilibrium condition $\mathbf{F}_{b}=0$ gives

$$
\mathbf{T}^{T} \mathbf{R}(\mathbf{T} \dot{\mathbf{q}}+\mathbf{E u})=0 .
$$

Finally, (19) can be inverted to obtain the relation between the swimming velocity $\dot{\mathbf{q}}$ and the input $\mathbf{u}$ as

$$
\dot{\mathbf{q}}=\mathbf{G}(\mathbf{q}) \mathbf{u}, \quad \text { where } \mathbf{G}(\mathbf{q})=\left(\mathbf{T}^{T} \mathbf{R T}\right)^{-1} \mathbf{T R E}
$$

Equation (20) describes the dynamics of the swimmer, formulated as a driftless nonlinear control system [13].

In the presence of an infinite wall at $y=0$, an important property of (20) is its invariance under shifting parallel to the wall, in the $x$ direction. That is, $\mathbf{G}(\mathbf{q})$ in (20) is independent of $x$ and depends only on the coordinates $y$ and $\theta$. Therefore, denoting $\mathbf{q}^{\prime}=(y, \theta)$, we define the reduced dynamics, which is given by

$$
\dot{\mathbf{q}}^{\prime}=\mathbf{G}^{\prime}\left(\mathbf{q}^{\prime}\right) \mathbf{u}
$$

where $\mathbf{G}^{\prime}$ is the lower $2 \times m$ block of $\mathbf{G}(\mathbf{q})$ in (20). In the next two sections, we study the properties of equilibrium points of (21) under fixed input $\mathbf{u}=\mathbf{u}_{e}$. Physically, these equilibria correspond to steady motion of the swimmer in pure translation parallel to the wall, with fixed orientation and distance from the wall $\mathbf{q}_{e}^{\prime}=\left(y_{e}, \theta_{e}\right){ }^{1}{ }^{1}$

Example 1. The two-sphere swimmer perpendicular to the wall. Consider the two spheres of radius $a=1$ near a wall, whose mobility matrix was given in (13) in the previous example. The spheres are now connected by a thin rigid rod of length $d$, and are actuated by rotation about the $z$-axis by angular velocities $u_{1}, u_{2}$ with respect to the rod (see Figure $2(\mathrm{~b})$ ). Assigning a reference frame $\mathcal{F}_{b}$ at the center of the rod, the motion of the swimmer is governed by (20). The closed-form expression for $\mathbf{G}(q)$ even for this simple model is extremely long and complicated. Therefore, we focus on the orientation $\theta=0$ for which the rod is perpendicular to the wall. In this case, the elements of $\mathbf{G}(\mathbf{q})$ are given by

$$
\mathbf{G}(\mathbf{q})=\left(\begin{array}{cc}
g_{11} & g_{12} \\
0 & 0 \\
g_{32} & g_{33}
\end{array}\right)
$$

\footnotetext{
${ }^{1}$ Mathematically, $\mathbf{q}_{e}^{\prime}$ is called a relative equilibrium [50] of (20), as it involves motion along $x$.
} 


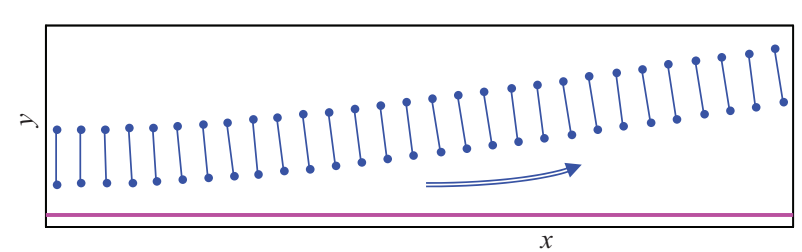

(a)

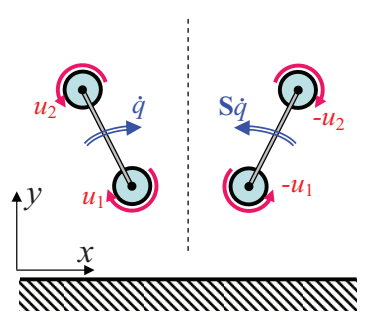

(b)

Figure 3. (a) Motion snapshots of the two-sphere swimmer near a wall with $u_{1}=-u_{2}$. (b) Illustration of the reversing symmetry.

where

$$
\begin{aligned}
& g_{11}=\frac{1}{w_{11} w_{33}-w_{13}^{2}}\left(w_{13}\left(r_{55}+r_{56}+\left(r_{15}-r_{35}\right) \frac{d}{2}\right)-w_{33}\left(r_{15}+r_{35}\right)\right), \\
& g_{12}=\frac{1}{w_{11} w_{33}-w_{13}^{2}}\left(w_{13}\left(r_{56}+r_{66}+\left(r_{16}-r_{36}\right) \frac{d}{2}\right)-w_{33}\left(r_{16}+r_{36}\right)\right), \\
& g_{31}=-\frac{1}{w_{11} w_{33}-w_{13}^{2}}\left(w_{11}\left(r_{55}+r_{56}+\left(r_{15}-r_{35}\right) \frac{d}{2}\right)-w_{13}\left(r_{15}+r_{35}\right)\right), \\
& g_{31}=-\frac{1}{w_{11} w_{33}-w_{13}^{2}}\left(w_{11}\left(r_{56}+r_{66}+\left(r_{16}-r_{35}\right) \frac{d}{2}\right)-w_{13}\left(r_{16}+r_{36}\right)\right), \\
& w_{11}=r_{11}+2 r_{13}+r_{33}, w_{13}=r_{15}+r_{16}+r_{35}+r_{36}+\left(r_{11}-r_{33} \frac{d}{2}\right), w_{22}=r_{22}+2 r_{24}+r_{44}, \\
& w_{33}=r_{55}+r_{56}+r_{66}+\left(r_{15}+r_{16}-r_{35}-r_{36}\right) d+\left(r_{11}-2 r_{13}+r_{33}\right) \frac{d^{2}}{4},
\end{aligned}
$$

and $r_{i j}$ is the $i j$-element of the resistance matrix $\mathbf{R}=\mathbf{M}^{-1}$, where $\mathbf{M}$ is given in (13). In the case of unbounded fluid, i.e., in the limit $h \rightarrow \infty$, the elements of $\mathbf{G}$ in (22) simplify to

$$
g_{12}=-g_{11}=\frac{d}{2 d^{3}+1}, \quad g_{31}=g_{32}=-2 \frac{8 d^{3}-9 d^{2}-4}{6 d^{5}+32 d^{3}-51 d^{2}-16} .
$$

Therefore, an input $\mathbf{u}$ of equal and opposite angular velocities $-u_{1}=u_{2}>0$ will result in pure translation motion of the swimmer in the positive $x$ direction, due to the axisymmetry of the configuration. This is consistent with the results in [47] for the axisymmetric twirling torus and its two-dimensional equivalent of two connected counter-rotating cylinders. However, the presence of a wall, i.e., $h<\infty$, destroys the axisymmetry, and under the same input $\mathbf{u}$ the swimmer rotates and deviates away from the wall. This is illustrated in Figure 3(a), which depicts snapshots of the swimmer's motion generated by numerical simulation of the two-sphere swimmer with $a=1, d=6$ under initial configuration $y=10$ and $\theta=0$ and input $\mathbf{u}=(-1,1)^{T}$. Motivated by this example, the rest of the paper will focus on studying the possibility of swimming in pure translation parallel to a wall and analyzing the dynamic stability of this motion under perturbations.

Copyright (C) by SIAM. Unauthorized reproduction of this article is prohibited. 
3. Dynamics of the two-sphere swimmer. In this section we consider the two-sphere swimmer model shown in Figure 2(b) and analyze the dynamics of its motion near a wall. An important observation is that this swimmer possesses fore-aft symmetry. That is, it has a reflection symmetry about the line connecting the centers of the two spheres. This symmetry implies the following key relation on the equation of swimming (20) under mirror reflection of the swimmer's configuration about the orientation perpendicular to the wall $\theta=0$, which is given by

$$
\mathbf{G}(\mathbf{S q})=-\mathbf{S G}(\mathbf{q}), \quad \text { where } \mathbf{S}=\operatorname{diag}(-1,1,-1) .
$$

The physical meaning of (23) is that a mirror-reflected swimmer will swim along a reflected trajectory under input velocities $\mathbf{u}$ with opposite sign (Figure 3(b)). Since reversing the input $\mathbf{u}$ is equivalent to reversing the time, a relation of the form (23) is formally called reversing symmetry of a dynamical system [44], where multiplication by the matrix $\mathbf{S}$ in (23) is called the reversing symmetry action. A direct consequence of (23) is that elements of the second row of $\mathbf{G}(\mathbf{q})$ are odd functions of $\theta$, while elements of the third row of $\mathbf{G}(\mathbf{q})$ are even functions of $\theta$, and hence they satisfy

$$
\begin{aligned}
g_{2 j}(\theta=0) & =0, \\
\left.\frac{\partial}{\partial y} g_{2 j}\right|_{\theta=0}=0,\left.\quad \frac{\partial}{\partial \theta} g_{3 j}\right|_{\theta=0} & =0
\end{aligned}
$$

for $j=1,2$, where $g_{i j}$ denotes the $(i, j)$-element of $\mathbf{G}(\mathbf{q})$. The fore-aft symmetry of the swimmer has a direct implication on the existence of steady translation parallel to the wall, as stated in the following theorem.

Theorem 3.1. Consider a planar low-Re swimmer with two inputs $\mathbf{u} \in \mathbb{R}^{2}$ whose motion is governed by (20), near an infinite no-slip wall located at $y=0$. Assume that the swimmer possesses fore-aft symmetry such that relation (23) holds. Then for any given distance from the wall $y=y_{e}$ there exists an input $\mathbf{u}_{e} \neq 0$ such that $\mathbf{q}_{e}^{\prime}=\left(y_{e}, 0\right)$ is an equilibrium point of the reduced dynamics (21) under the fixed input $\mathbf{u}=\mathbf{u}_{e}$.

Proof. The relation (24) implies that for $\theta=0$ the $\dot{y}$-component of $\dot{\mathbf{q}}$ in (20) vanishes for any $y$ and $\mathbf{u}$, as seen in the structure of $\mathbf{G}(\mathbf{q})$ in (22). Therefore, for given distance from the wall $y=y_{e}$, taking input velocities $\mathbf{u}_{e}=\alpha\left(-g_{32}, g_{31}\right)$ where $g_{31}, g_{32}$ are evaluated at $(y, \theta)=\left(y_{e}, 0\right)$ and $\alpha \in \mathbb{R}$, the $\dot{\theta}$-component of $\dot{\mathbf{q}}$ also vanishes, and $\dot{\mathbf{q}}$ has the form $\dot{\mathbf{q}}=\left(v_{x}, 0,0\right)$, so that $\dot{y}=\dot{\theta}=0$, which completes the proof.

The theorem implies that for the two-sphere swimmer, steady translation parallel to the wall at the orientation perpendicular to the wall $\theta=0$ is always possible by appropriately choosing the input $\mathbf{u}$. The physical meaning of the chosen input $\mathbf{u}_{e}$ is as follows. When the two-sphere swimmer is placed near a wall, the sphere closer to the wall experiences larger hydrodynamic resistance than the other sphere. Therefore, rotating the spheres at equal and opposite angular velocities $u_{1}=-u_{2}$ generates a nonzero net torque about the rod's center, which must be canceled by rigid-body rotation of the swimmer, as illustrated in Figure 3(a). Nevertheless, for each separation distance from the wall there exists a specific ratio of input velocities $u_{1}$ and $u_{2}$ that compensates for the difference in resistances by rotating the sphere

Copyright (c) by SIAM. Unauthorized reproduction of this article is prohibited. 


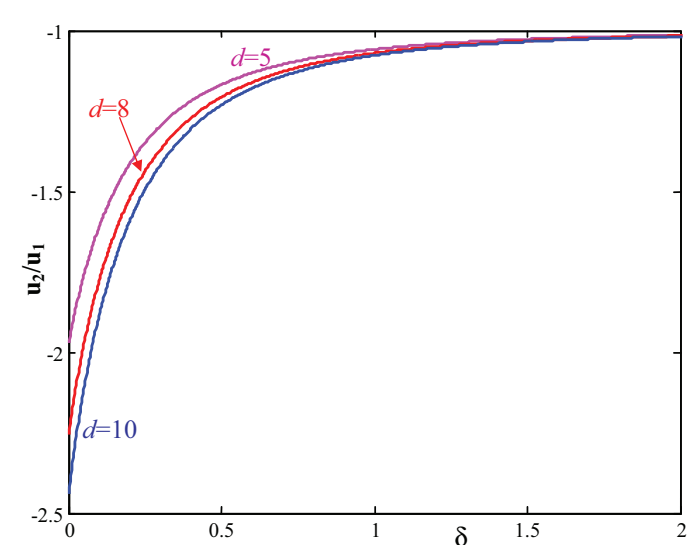

(a)

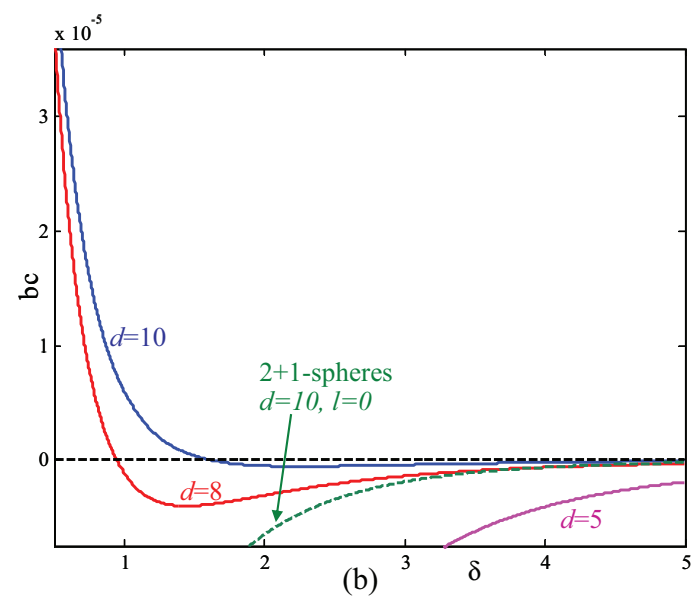

(b)

Figure 4. (a) The ratio $u_{2} / u_{1}$ for parallel translation as a function of $\delta$. (b) The product bc as a function of $\delta$.

closer to the wall more slowly. In that case, the resulting net torque is zero under pure translation. As an example, Figure 4(a) plots the ratio $u_{2} / u_{1}$ which is required in order to maintain translation parallel to the wall at a distance $\delta=h-a$ from the wall as a function of $\delta$, for the two-sphere swimmer with $a=1$ and three different values of the distance $d$ between the spheres. It can be seen that $u_{2} / u_{1}$ is always negative; that is, the spheres are counterrotating. (If the two spheres rotate in the same direction, the swimmer's body must rotate in the opposite direction in order to balance the net torque.) Moreover, $\left|u_{2} / u_{1}\right|$ is increasing when $\delta$ is small, and it converges to 1 as $\delta \rightarrow \infty$, which is precisely the case of straight-line swimming in unbounded fluid under equal and opposite angular velocities $u_{1}$ and $u_{2}$.

3.1. Dynamic stability of the two-sphere swimmer near a wall. We now analyze the dynamic stability of parallel translation near the wall for the two-sphere swimmer with fixed input $\mathbf{u}=\mathbf{u}_{e}$. This is equivalent to characterizing the stability of the equilibrium point $\mathbf{q}_{e}^{\prime}=\left(y_{e}, 0\right)$ of $(21)$, which is summarized in the following theorem.

Theorem 3.2. Consider a planar low-Re swimmer with two inputs $\mathbf{u} \in \mathbb{R}^{2}$, whose motion is governed by (20), near an infinite no-slip wall located at $y=0$. Assume that the swimmer possesses fore-aft symmetry such that relation (23) holds. For a given input $\mathbf{u}=\mathbf{u}_{e}$ and the corresponding equilibrium point $\mathbf{q}_{e}^{\prime}=\left(y_{e}, 0\right)$ of (21), define

$$
\begin{aligned}
& b=\left.\left(g_{31} \cdot \frac{\partial g_{22}}{\partial \theta}-g_{32} \cdot \frac{\partial g_{21}}{\partial \theta}\right)\right|_{\mathbf{q}^{\prime}=\mathbf{q}_{e}^{\prime}}, \\
& c=\left.\left(g_{31} \cdot \frac{\partial g_{32}}{\partial y}-g_{32} \cdot \frac{\partial g_{31}}{\partial y}\right)\right|_{\mathbf{q}^{\prime}=\mathbf{q}_{e}^{\prime}},
\end{aligned}
$$

where $g_{i j}$ is the $(i, j)$-element of $\mathbf{G}(\mathbf{q})$ in (20). Then the dynamic stability of $\mathbf{q}_{e}^{\prime}$ under fixed input is characterized as follows:

- If bc $>0$, then $\mathbf{q}_{e}^{\prime}$ is unstable.

- If $b c<0$, then $\mathbf{q}_{e}^{\prime}$ is marginally stable. Moreover, under sufficiently small initial perturbations about $\mathbf{q}_{e}^{\prime}$, the solution of (21) is periodic. 
Proof. The local stability of $\mathbf{q}_{e}^{\prime}$ is determined by the linearization of (21) about $\mathbf{q}_{e}^{\prime}$, given by

$$
\delta \dot{\mathbf{q}}^{\prime}=\mathbf{A} \delta \mathbf{q}^{\prime}, \quad \text { where } \mathbf{A}=\left.\frac{\partial \mathbf{G}^{\prime}\left(\mathbf{q}^{\prime}\right)}{\partial \mathbf{q}^{\prime}}\right|_{\mathbf{q}_{e}^{\prime}} \cdot \mathbf{u}_{e} .
$$

The classical Hartman-Grobman theorem (cf. [31]) implies that a sufficient condition for asymptotic stability of $\mathbf{q}_{e}^{\prime}$ is that all eigenvalues of $\mathbf{A}$ have negative real parts. However, invoking the constraints (25) and substituting $\mathbf{u}_{e}=\alpha\left(-g_{32}, g_{31}\right)$, the linearization matrix $\mathbf{A}$ takes the form

$$
\mathbf{A}=\alpha\left(\begin{array}{ll}
0 & b \\
c & 0
\end{array}\right)
$$

where $b$ and $c$ are defined in (26). The characteristic polynomial of $\mathbf{A}$ for $\alpha=1$ is $\Delta_{A}(\lambda)=$ $\lambda^{2}-b c$. The eigenvalues of $\mathbf{A}$ are thus a symmetric pair ${ }^{2} \lambda= \pm \sqrt{b c}$, which can be either real or purely imaginary, depending on the sign of $b c$. In the case where $b c>0$, the two eigenvalues of $\mathbf{A}$ are a real pair, one of which is positive, indicating that $\mathbf{q}_{e}^{\prime}$ is an unstable saddle point. In the case where $b c<0$, the two eigenvalues of $\mathbf{A}$ form a purely imaginary pair having zero real part. In general, this fact is not sufficient for deducing stability or instability of $\mathbf{q}_{e}^{\prime}$. Nevertheless, the reversing symmetry imposes extra structure on the system, as follows. Since $\theta=0$ is the "mirror axis" of reflection, the equilibrium point $\mathbf{q}_{e}^{\prime}$ lies on the fixed set of the reversing symmetry, that is, $\mathbf{q}_{e}^{\prime}=\mathbf{S}^{\prime} \mathbf{q}_{e}^{\prime}$, where $\mathbf{S}^{\prime}=\operatorname{diag}(1,-1)$ is the $(y, \theta)$-component of the reversing symmetry $\mathbf{S}$ in (23). In this case, a classical theorem by Devaney [19, 44] states that $\mathbf{q}_{e}^{\prime}$ is a reversible Lyapunov center, which is a marginally stable equilibrium point enclosed by a one-parameter family of periodic orbits.

According to the theorem, the stability characterization of $\mathbf{q}_{e}^{\prime}$ strongly depends on the sign of the product $b c$, defined in (26). In order to compute $b c$, the partial derivatives of the matrix $\mathbf{G}$ with respect to the coordinates $y$ and $\theta$ must be computed explicitly. These derivatives can be obtained by applying the chain rule on the definition of $\mathbf{G}(\mathbf{q})$ in $(20)$, and are given by

$$
\begin{aligned}
& \frac{\partial \mathbf{G}}{\partial y}=\mathbf{M}_{b} \mathbf{T}^{T} \mathbf{R} \frac{\partial \mathbf{M}}{\partial y} \mathbf{R}\left(\mathbf{I}-\mathbf{T} \mathbf{M}_{b} \mathbf{T}^{T} \mathbf{R}\right) \mathbf{E} \\
& \frac{\partial \mathbf{G}}{\partial \theta}=\mathbf{M}_{b}\left(\left(\mathbf{T}^{T} \mathbf{R} \frac{\partial \mathbf{M}}{\partial \theta}-\frac{\partial \mathbf{T}^{T}}{\partial \theta}\right) \mathbf{R}\left(\mathbf{I}-\mathbf{T} \mathbf{M}_{b} \mathbf{T}^{T} \mathbf{R}\right)+\mathbf{T}^{T} \mathbf{R} \frac{\partial \mathbf{T}}{\partial \theta} \mathbf{M}_{b} \mathbf{T}^{T} \mathbf{R}\right) \mathbf{E},
\end{aligned}
$$

where $\mathbf{M}_{b}=\left(\mathbf{T}^{T} \mathbf{R T}\right)^{-1}$ and $\mathbf{I}$ is the identity matrix. The derivatives of the mobility matrix $\mathbf{M}=\mathbf{R}^{-1}$ in (28) are obtained by direct differentiation of the expressions in [68]. Using these expressions, the terms $b$ and $c$ in (26) were obtained, and their product was numerically computed. Figure 4(b) shows a plot of $b c$ as a function of the equilibrium distance from the wall $\delta$, for $a=1$ and three different values of the center-center distance $d$. The plot reveals that when $\delta$ is greater than a lower bound, the product $b c$ is negative. Thus, according to Theorem $3.2, \mathbf{q}_{e}^{\prime}$ is a marginally stable equilibrium point enclosed by a one-parameter family of periodic orbits.

\footnotetext{
${ }^{2}$ This is typical of systems with reversing symmetry [44], for which the existence of a solution of (27) that evolves in time as $e^{\lambda t}$ implies the existence of a reflected and time-reversed solution evolving as $e^{-\lambda t}$.
} 


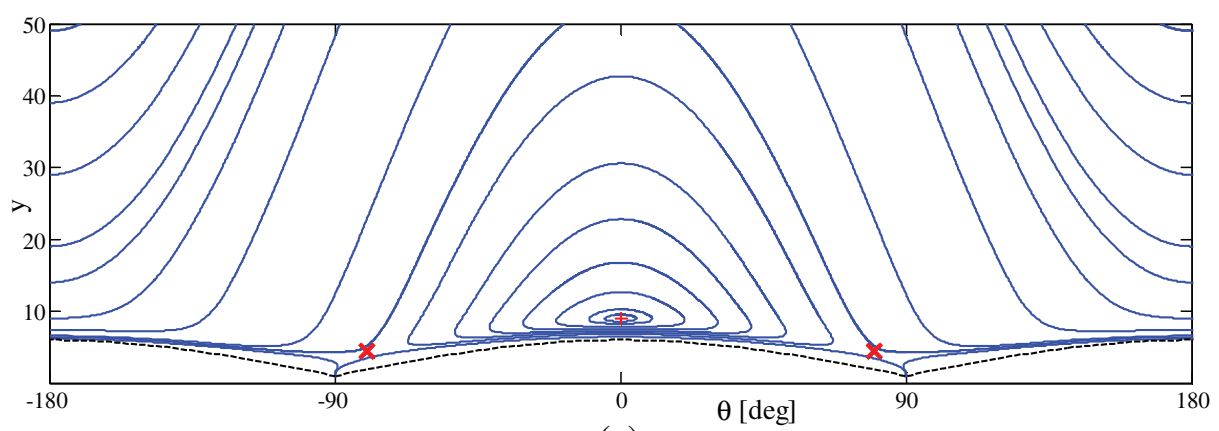

(a)

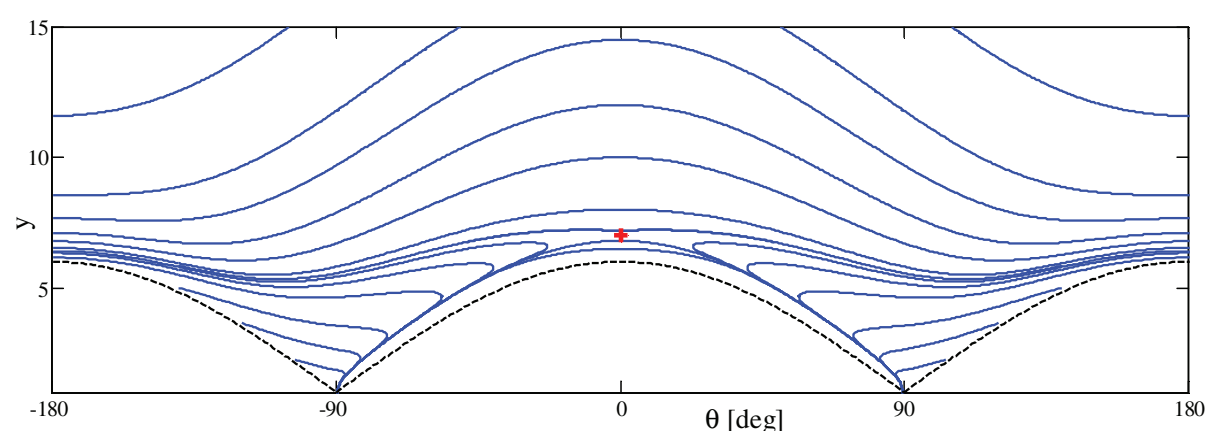

(b)

Figure 5. Phase portrait of trajectories of the two-sphere swimmer in the $(\theta, y)$-plane with $a=1$ and $d=10$ for (a) $u_{2} / u_{1}=-1.0068$ and (b) $u_{2} / u_{1}=-1.0774$.

Example 2. Phase portraits of the two-sphere swimmer dynamics near a wall. Consider the two-sphere swimmer with $a=1, d=10$, and input $\mathbf{u}_{e}=(1,-1.0068)$, which corresponds to an equilibrium point $\mathbf{q}_{e}^{\prime}=\left(9,0^{\circ}\right)$, that is, $\delta=3$. The two eigenvalues of the linearization of (21) about $\mathbf{q}_{e}^{\prime}$ are $\pm 0.77 i \times 10^{-3}$ (since $b c<0$; see Figure $4(\mathrm{~b})$ ), so that $\mathbf{q}_{e}^{\prime}$ is a Lyapunov center. Figure 5(a) plots the phase portrait of solution trajectories of $(21)$ in the $(\theta, y)$-plane under fixed input $\mathbf{u}=\mathbf{u}_{e}$. The dashed curve corresponds to configurations at which one of the spheres touches the wall; thus they bound the physically permissible region in the $(\theta, y)$-plane. The solid curves are solution trajectories of $(\theta, y)$ under different initial conditions. ${ }^{3}$ It can be seen that the equilibrium point $\mathbf{q}_{e}^{\prime}=\left(0^{\circ}, 9\right)$ (marked by "+" in the figure) is enclosed by a family of closed curves. These curves represent periodic $(\theta, y)$-solutions under small perturbations about $\mathbf{q}_{e}^{\prime}$, which correspond to oscillating motion along the wall, as shown in Figure 6(a). When the initial perturbation about $\mathbf{q}_{e}^{\prime}$ is large, a different type of solution trajectories emerge, which no longer form closed curves around $\mathbf{q}_{e}^{\prime}$. These curves correspond to cycloid-like trajectory along the wall, as shown in Figure 6(b), under which the swimmer's orientation $\theta(t)$ grows monotonically. Additional off-symmetric equilibrium points also exist at $\theta= \pm 79.85^{\circ}$, marked by " $\times$ " in Figure 5 . These are unstable saddle points. The separatrix curves which separate the two types of solution curves (oscillating and spiralling motion) are trajectories that emanate from (and converge to) these saddle points.

\footnotetext{
${ }^{3}$ Note that the phase portrait is $360^{\circ}$-periodic in $\theta$ and has a reflection symmetry about the lines $\theta=0^{\circ}$ and $\theta=180^{\circ}$, as implied by the reversing symmetries of the system.
}

Copyright (C) by SIAM. Unauthorized reproduction of this article is prohibited. 

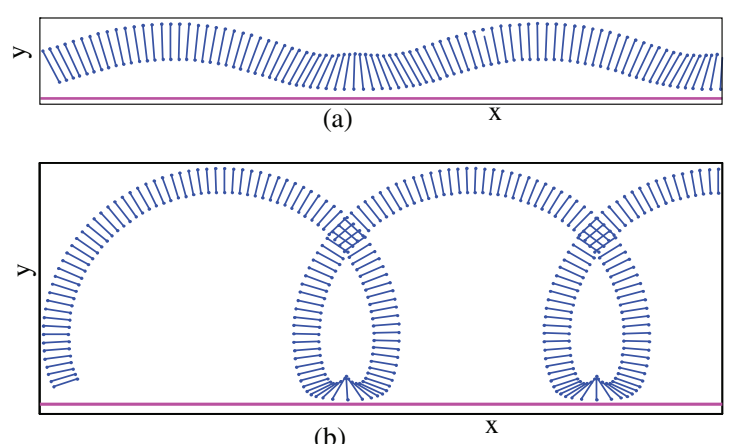

(b)

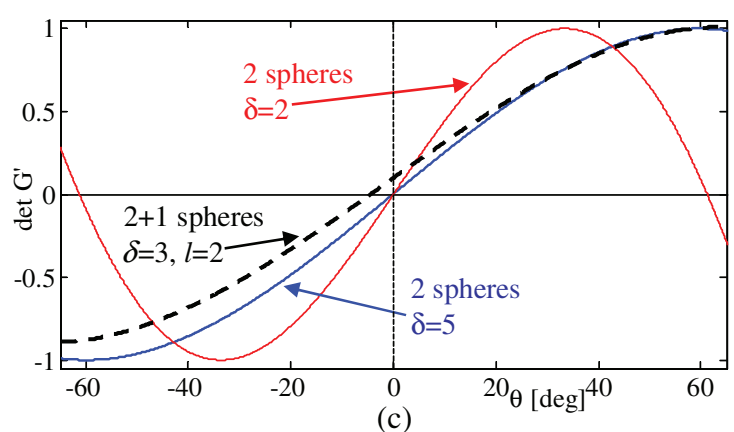

(c)

Figure 6. (a) Periodic oscillations near the wall. (b) Spiral motion near the wall. (c) $\operatorname{det}^{\prime} \mathbf{G}^{\prime}$ as a function of $\theta$.

As a second example, consider the same swimmer under fixed input $\mathbf{u}_{e}=(1,-1.0774)$, which corresponds to an equilibrium point $\mathbf{q}_{e}^{\prime}=\left(0^{\circ}, 7\right)$, that is, $\delta=1$. Figure 5 (b) plots the phase portrait of solution trajectories of $(21)$ in the $(\theta, y)$-plane. In this case, $\mathbf{q}_{e}^{\prime}$ is an unstable equilibrium with corresponding linearization eigenvalues of \pm 0.0018 (since $b c>0$; see Figure 4(b)). Thus, any perturbation about the equilibrium point $\mathbf{q}_{e}^{\prime}$ (which corresponds to steady parallel translation along the wall) results in solutions of cycloid-like trajectories similar to those shown in Figure 6(b).

The above analysis shows that translation parallel to the wall for the two-sphere swimmer in the perpendicular orientation $\theta=0$ cannot be asymptotically stable. In the following, we seek a steady parallel translation which corresponds to equilibrium points of (21) for different orientations $\theta \neq 0$. A necessary condition for existence of an equilibrium point of (21) under constant input $\mathbf{u}=\mathbf{u}_{e}$ is that the columns of the matrix $\mathbf{G}^{\prime}\left(\mathbf{q}^{\prime}\right)$ are linearly dependent, that is, $\operatorname{det} \mathbf{G}^{\prime}=0$. We numerically compute $\operatorname{det} \mathbf{G}^{\prime}$ as a function of the orientation $\theta$ for the two-sphere swimmer with $d=10$, while the wall-separation $\delta$ is held constant. The results are shown in Figure $6(\mathrm{c})$ for $\delta=5$ and $\delta=2$. It can be seen that for large separations such as $\delta=5$, the only zero-crossing is at $\theta=0$, whereas for the smaller separation $\delta=2$, there exists an additional zero-crossing point at $\theta=61.25^{\circ}$, associated with input vector $\mathbf{u}_{e}=(1,-1.017)^{T}$. Numerical computation of the linearization matrix $\mathbf{A}$ and its eigenvalues gives $\lambda_{1}=-0.0012, \lambda_{2}=0.0008$; hence the equilibrium point $\mathbf{q}_{e}^{\prime}$ is unstable. Similar results which were obtained numerically for other values of $d$ and $\delta$ indicate that these additional equilibrium point are always unstable and correspond to the saddle points appearing in the phase portrait of Figure 5(a) (marked by " $\times$ ").

4. Dynamics of the 2+1-sphere swimmer. In this section we study the model of the $2+1$-sphere swimmer, shown in Figure 2(c). This model is an extension of the two-sphere swimmer, in which an additional unactuated sphere is rigidly attached to the swimmer's body. The three spheres form an isosceles triangle with height $l$, as shown in Figure 2(c). First, consider the case $l=0$ for which the centers of the three spheres are collinear. In this special case, the property of reversing symmetry in (23) also holds for the $2+1$-sphere swimmer. Therefore, Theorem 3.1 implies the existence of steady motion parallel to the wall, with an equilibrium point $\mathbf{q}_{e}^{\prime}=\left(y_{e}, 0\right)$ of $(21)$ under a constant input $\mathbf{u}=\mathbf{u}_{e}$. Moreover, 

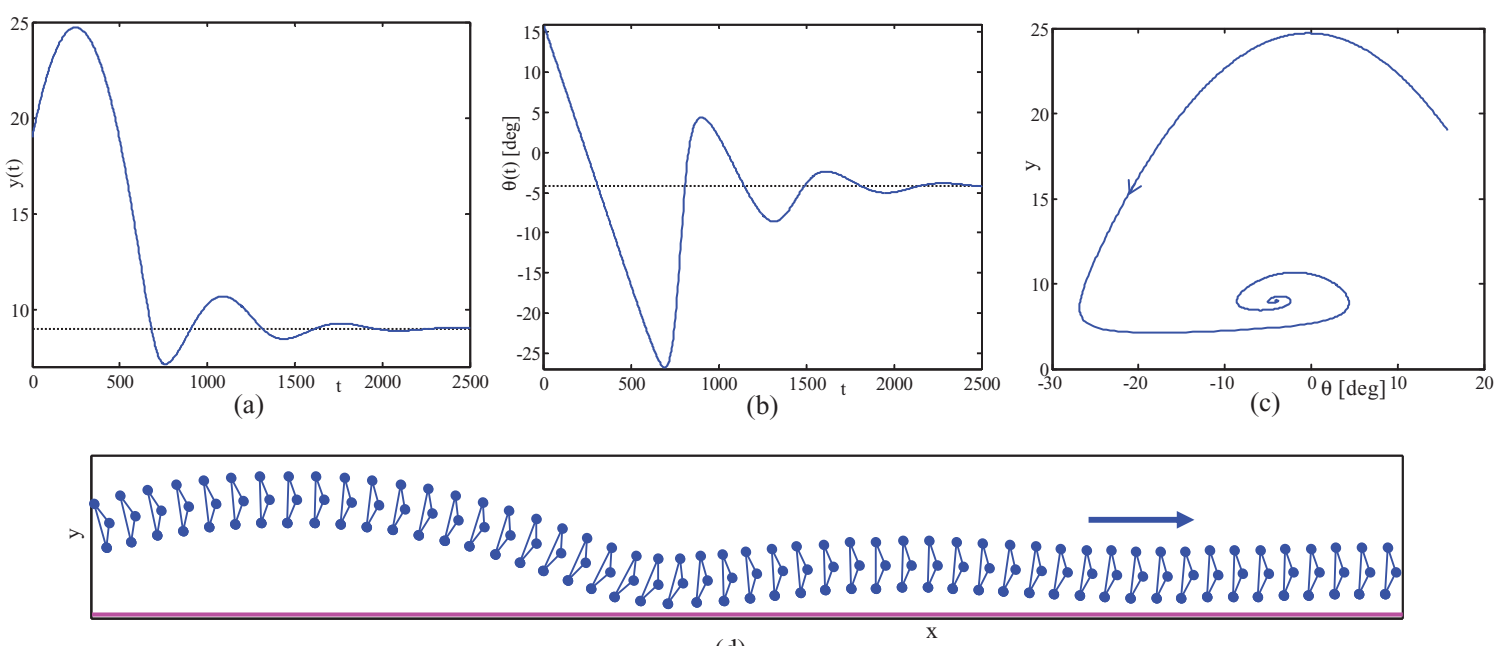

(d)

Figure 7. Simulation of the 2+1-spheres swimmer under constant input. (a) Plot of $y(t)$. (b) Plot of $\theta(t)$. (c) Trajectory in $(\theta, y)$-plane. (d) Snapshots of swimmer's motion near the wall.

according to Theorem 3.2, the stability of $\mathbf{q}_{e}^{\prime}$ is determined by the sign of $b c$, defined in (26). The product $b c$ was numerically computed for the $2+1$-sphere swimmer with $a=1, d=10$, and $l=0$ as a function of the distance from the wall $\delta$. The result is shown in the dashed curve in Figure 4(b) and indicates that $b c<0$ for any distance $\delta>0$. According to Theorem 3.2, this implies that $\mathbf{q}_{e}^{\prime}$ is again a reversible Lyapunov center and that the dynamic trajectories of the swimmer are similar to the phase portrait of Figure 5(a).

Next, we consider the general case of $l \neq 0$. In this case, the reversing symmetry relation (23) does not hold. Therefore, swimming parallel to the wall at $\theta=0$ is generally impossible. Nevertheless, it can be possible under different orientations $\theta \neq 0$. To illustrate this, we regard $l$ as a parameter of the system which is changed continuously from zero. Consider again the computation of $\operatorname{det} \mathbf{G}^{\prime}$ as a function of $\theta$ for a fixed distance $\delta$ from the wall. For the swimmer with $l=0, \operatorname{det} \mathbf{G}^{\prime}$ vanishes at $\theta=0$ due to the reversing symmetry. Under a small change in $l$, $\operatorname{det} \mathbf{G}^{\prime}$ changes continuously, and its zero crossing point shifts from $\theta=0$ to some nonzero $\theta$. As an example, $\operatorname{det} \mathbf{G}^{\prime}$ for the $2+1$-sphere swimmer with $d=10, l=2$, and $\delta=3$ is shown in the dashed curve in Figure $6(\mathrm{c})$. For these values, $\operatorname{det} \mathbf{G}^{\prime}$ vanishes at $\theta=-4.19^{\circ}$. This corresponds to an equilibrium point $\mathbf{q}_{e}^{\prime}=\left(8.987,-4.19^{\circ}\right)$ of $(21)$ under the input $\mathbf{u}_{e}=(1,1.0086)$. Moreover, since the equilibrium point $\mathbf{q}_{e}^{\prime}$ changes continuously with $l$, the eigenvalues of the linearization matrix $\mathbf{A}$ about $\mathbf{q}_{e}^{\prime}$ in (27) also change continuously. For $l=0$ the two eigenvalues are a purely imaginary pair $\lambda= \pm i \omega_{0}$ (since $b c<0$ ). Thus, under a small change in $l$ they change to a complex conjugate pair $\lambda=\sigma \pm i \omega$. From the definition of $\mathbf{A}$ in (27), it is clear that the eigenvalues scale linearly with multiplying $\mathbf{u}_{e}$ by a scalar $\alpha$. Therefore, the sign of $\alpha$ can be appropriately chosen in order to guarantee that $\sigma<0$, leading to asymptotic stability of $\mathbf{q}_{e}^{\prime}$. As a numerical example, we simulate the dynamics of the $2+1$-sphere swimmer with $d=10$ and $l=2$ under the fixed input $\mathbf{u}_{e}=(1,1.0086)$, corresponding to the equilibrium point $\mathbf{q}_{e}^{\prime}=\left(8.987,-4.19^{\circ}\right)$ with linearization eigenvalues of $(-0.3984 \pm 1.5341 i) \cdot 10^{-3}$. Figure $7(\mathrm{a})-(\mathrm{b})$ shows simulation results of $y(t)$ and $\theta(t)$ for this 


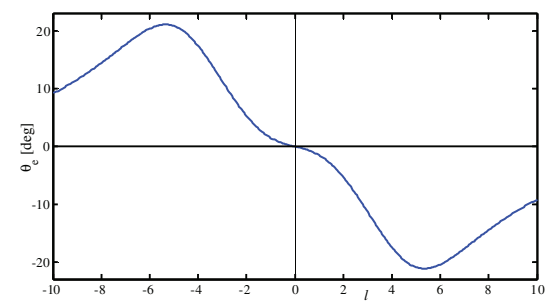

(a)

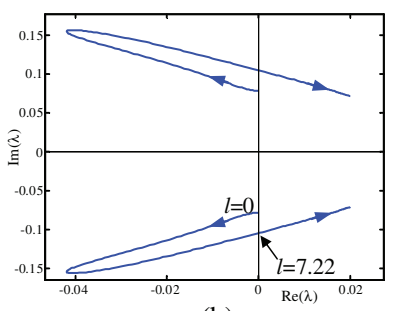

(b)

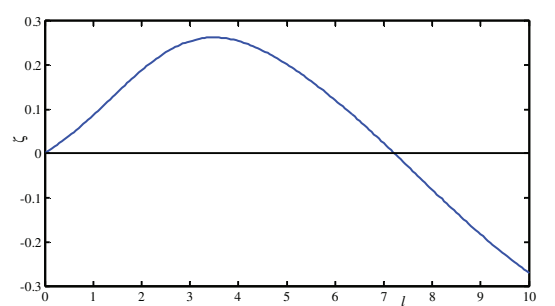

(c)

Figure 8. (a) The equilibrium orientation $\theta_{e}$ as a function of $l$ for the $2+1$-spheres swimmer. (b) Locus of the linearization eigenvalues in complex plane for $l \in[0,10]$. (c) The damping ratio $\zeta$ as a function of $l$.

swimmer under initial perturbation of $\mathbf{q}^{\prime}(0)=\mathbf{q}_{e}^{\prime}+\left(10,20^{\circ}\right)$. Figure $7(\mathrm{c})$ plots the trajectory in the $(\theta, y)$-plane, and Figure $7(\mathrm{~d})$ shows snapshots of the swimmer's motion along the wall. The plots clearly indicate that $\mathbf{q}_{e}^{\prime}$ is asymptotically stable and that the swimmer converges to steady translation parallel to the wall.

It is important to note that the stability result is not necessarily limited to the specific model of the $2+1$-sphere swimmer. Any low-Re swimmer with a fixed shape whose nominal structure satisfies the reversing symmetry relation (23) can break its fore-aft symmetry properly in order to be able to track a straight wall with asymptotic stability. It is also remarkable that this stabilization is achieved passively (or in "open loop") without incorporating any onboard position sensing or feedback control. The stabilization here is a purely hydrodynamic effect due to the swimmer's geometry and its interaction with the wall.

Next, we numerically study the influence of the strength of the fore-aft symmetry breaking on the stability of swimming parallel to the wall. This is done by considering the $2+1$-sphere swimmer with $d=6$ and $a=1$ while the symmetry-breaking parameter $l$, regarded here as a signed distance, varies in the range $[-10,10]$. For a fixed separation distance $\delta=2$, we numerically compute the orientation angle $\theta_{e}$ of the swimmer at equilibrium configuration $\mathbf{q}_{e}^{\prime}$ (by finding the zero crossing of $\mathbf{d e t} \mathbf{G}^{\prime}$ ) and the corresponding input $\mathbf{u}_{e}$. The magnitude of $\mathbf{u}_{e}$ is then chosen such that the swimmer moves in unit velocity along the positive $x$ direction. The eigenvalues of the linearization matrix of (21) about $\mathbf{q}_{e}^{\prime}$ are derived by computing the matrix $\mathbf{A}$ in (27) according to (28). Figure 8(a) plots $\theta_{e}$ as a function of $l$. It can be seen that $\theta_{e}$ is an odd function of $l$, since the swimmer possesses an additional reflection symmetry with respect to reversing the sign of $l$. Figure 8(b) shows the locus of the two eigenvalues of the linearization matrix $\mathbf{A}$ in the complex plane while $l$ varies within the range $[0,10]$. It can be seen that for $l=0$ the eigenvalues are purely real, then they move to the left half plane for $l>0$; i.e., $\mathbf{q}_{e}^{\prime}$ becomes asymptotically stable. Then at $l=7.22$ the eigenvalues cross the imaginary axis again, and $\mathbf{q}_{e}^{\prime}$ becomes unstable for $l>7.22$. For the range $l<0$, which is not shown in the figure, the eigenvalues simply reverse their signs, and the stability characterization is reversed. In order to quantify the stability of $\mathbf{q}_{e}^{\prime}$, a natural measure is the damping ratio, defined as $\zeta(\sigma \pm i \omega)=-\sigma / \sqrt{\sigma^{2}+\omega^{2}}$, which corresponds to the nondimensional rate of convergence to $\mathbf{q}_{e}^{\prime}$. Figure 8(c) plots $\zeta$ as a function of $l$, indicating that $\zeta$ attains a maximal value at $l \approx 3.5$, where $\mathbf{q}_{e}^{\prime}$ is "most stable."

4.1. Periodic motion of the $2+1$-sphere swimmer. The $2+1$-sphere swimmer with a varying parameter $l$ can be interpreted as a family of dynamical systems parameterized by 


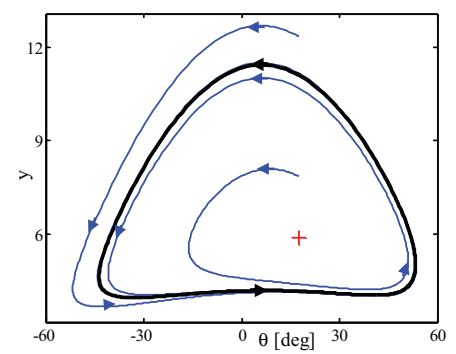

(a)

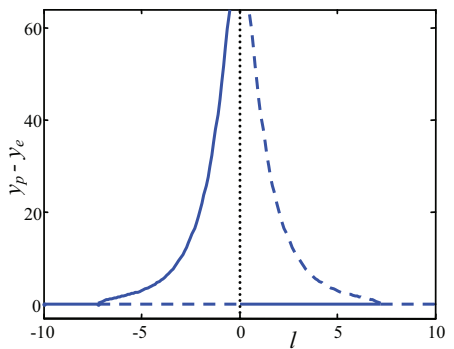

(c)

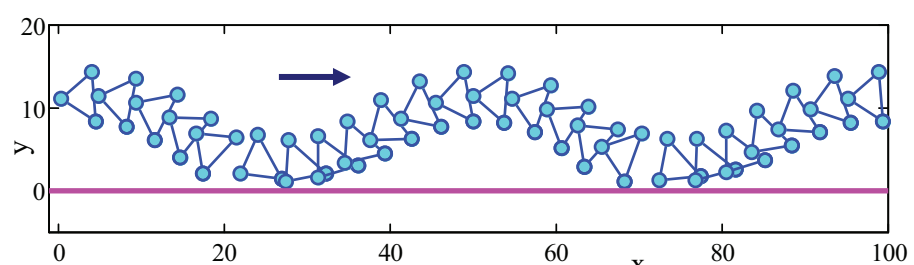

(b)

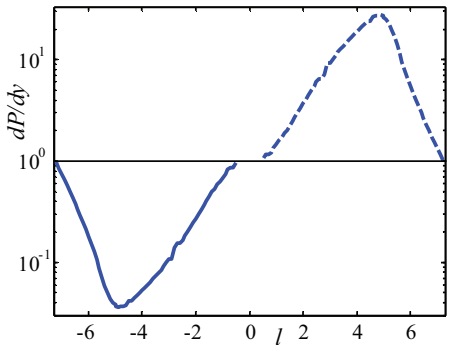

(d)

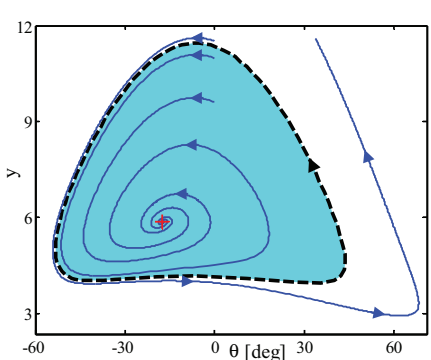

(e)

Figure 9. (a) Phase portrait of a stable periodic orbit for the $2+1$-spheres swimmer. (b) Snapshots of the periodic motion along the wall. (c) Amplitude of a periodic orbit $y_{p}-y_{e}$ as a function of $l$. (d) Derivative of the Poincaré map $\mathcal{P}$ as a function of $l$. (e) Phase portrait of a stable equilibrium and its region of attraction.

$l$. The changes in the equilibrium point $\mathbf{q}_{e}^{\prime}$ and its stability characterizations are described in Figure 8. It can be seen in Figure 8(b) that the eigenvalues cross the imaginary axis for some critical values of $l$. These values are associated with a Hopf bifurcation of the system [29] and indicate the possible evolution of periodic solutions of (21) once $l$ crosses a critical value. As an example, we simulate the dynamics of the $2+1$-cylinder swimmer for $a=1$, $r=6$, and $l=-4$, under input $\mathbf{u}_{e}=(-1,1.0605)$ corresponding to an equilibrium point $\mathbf{q}_{e}^{\prime}$ with $\delta=2$, which is unstable. Figure 9 (a) plots solution trajectories in the $(\theta, y)$-plane under different initial conditions. The phase portrait clearly shows the existence of a periodic orbit, which is associated with oscillations along the wall, as shown in the snapshots of Figure 9(b). Moreover, the plot shows that this periodic orbit is locally stable, in the sense that under small initial perturbations the solution converges back to this orbit.

In order to conduct a parametric study and stability characterization of periodic solutions, we use the notion of a Poincaré map, defined briefly as follows. Consider a dynamical system $\dot{\mathbf{x}}=f(\mathbf{x})$, where $\mathbf{x} \in \mathbb{R}^{n}$. A Poincaré section $\mathcal{S} \subset \mathbb{R}^{n}$ is a smooth manifold of dimension $n-1$ which is transversal to solution trajectories of the dynamical system. Next, we define the Poincaré map $\mathcal{P}: \mathcal{S} \rightarrow \mathcal{S}$ as $\mathcal{P}\left(s_{0}\right)=\mathbf{x}(t=T)$, where $\mathbf{x}(t)$ is the solution of $\dot{\mathbf{x}}=f(\mathbf{x})$ under initial condition $\mathbf{x}(0)=s_{0}$ and $T=\min \{t>0: \mathbf{x}(t) \in \mathcal{S}\}$. That is, the Poincaré map simply tells where the solution under initial conditions $s_{0} \in \mathcal{S}$ intersects the Poincaré section $\mathcal{S}$ again for the first time. A fixed point $s_{p}$ of the Poincaré map which satisfies $\mathcal{P}\left(s_{p}\right)=s_{p}$ simply represents the starting point of a periodic orbit of the dynamical system. Moreover, the stability of a periodic orbit that passes through $s_{p}$ is equivalent to the stability of the fixed point $s_{p}$ of the discrete-time dynamical system $s_{k+1}=\mathcal{P}\left(s_{k}\right)$, which, in turn, is determined by the eigenvalues of the Jacobian matrix $[\partial \mathcal{P} / \partial s]$ evaluated at $s=s_{p}$. Specifically, the periodic 
orbit is locally stable if and only if the magnitude of all eigenvalues of the Jacobian matrix is less than one. ${ }^{4}$

In the case of the $2+1$-cylinder swimmer, under a given value of $l$, the dynamical system is given in (21), under the fixed input $\mathbf{u}_{e}$ which is associated with a given equilibrium point $\mathbf{q}_{e}^{\prime}=\left(y_{e}, \theta_{e}\right)$. We define the Poincaré section as $\mathcal{S}=\left\{(y, \theta): \quad \theta=\theta_{e}\right.$ and $\left.y \geq y_{e}\right\} . \mathcal{S}$ is a onedimensional manifold which can be simply parametrized by the $y$-component of its points. A periodic orbit is represented by a scalar value $y_{p}$ such that $\mathcal{P}\left(y_{p}\right)=y_{p}$. The condition for stability of a periodic orbit simply reduces to the scalar inequality $\left|d \mathcal{P} / d y\left(y=y_{p}\right)\right|<1$. Computation of the Poincaré map, searching for its fixed points, and computing its Jacobian are done numerically by integration of (21) using MATLAB. As an example, consider the $2+1-$ sphere swimmer with $a=1, d=6, \delta=2$, and $l$ varying within the range $[-10,10]$. Figure 9 (c) plots $y_{p}-y_{e}$ as a function of $l$. The horizontal line $y_{p}=y_{e}$ represents the equilibrium solution $\mathbf{q}^{\prime}=\mathbf{q}_{e}^{\prime}$. The additional curve with $y_{p}>y_{e}$ represents co-existing periodic solutions and also serves as a measure for the amplitude of the periodic oscillations about $\mathbf{q}_{e}^{\prime}$. Due to the reversing symmetry with respect to $l$, the plot is symmetric about $l=0$. The derivative of the Poincaré map $\lambda=d \mathcal{P} / d y$ evaluated at $y=y_{p}$ is shown in the semilogarithmic plot of Figure 9(d). The plot is antisymmetric with respect to $l$; that is, when the sign of $l$ is reversed, $l \rightarrow-l$, the derivative $\lambda$ is inverted $\lambda \rightarrow 1 / \lambda$ so that the stability characterization is reversed. Combining the information from this plot with the knowledge of the eigenvalues associated with linearization about the equilibrium $\mathbf{q}_{e}^{\prime}$ as shown in Figure 8(b)-(c) gives a complete stability characterization of the equilibrium solutions and the periodic orbits. The solid pieces of the line $y_{p}=y_{e}$ and the curve with $y_{p}>y_{e}$ in Figure 9 (c) represent stable equilibrium points and stable periodic orbits, respectively, while the dashed pieces represent unstable solutions.

We now briefly discuss the characterization of the dynamics of the $2+1$-sphere swimmer by interpreting the plot in Figure $9(\mathrm{c})$ as a bifurcation diagram. For $l \in(0,7.22)$, there exists a stable equilibrium point $\mathbf{q}_{e}^{\prime}$ and an unstable periodic orbit represented by $y_{p}$. It is important to note that this periodic orbit precisely encloses the region of attraction of the equilibrium point $\mathbf{q}_{e}^{\prime}$ in $(\theta, y)$-plane. That is, all initial conditions $(\theta(0), y(0))$ that lie inside the region enclosed by the unstable closed orbit guarantee convergence of the solution to $\mathbf{q}_{e}^{\prime}$. This is illustrated by the phase portrait of solution trajectories for $l=4$ in Figure 9(e). The shaded area enclosed by the unstable periodic orbit (shown as a dashed curve) is the region of attraction where solution trajectories converge to the stable equilibrium point $\mathbf{q}_{e}^{\prime}$ (marked by "+"). Note that this phase portrait is precisely a mirror reflection of the phase portrait in 9 (a) for $l=-4$ about the line $\theta=0$, while the direction of the trajectories is also reversed. When $l$ approaches the critical value of 7.22 , the amplitude of the periodic solution decreases to zero, making it coincide with the equilibrium solution. The associated eigenvalue of the Poincaré map approaches unity, leading to instability of the solution. For $l>7.22$, the periodic solution disappears and the equilibrium solution $\mathbf{q}^{\prime}=\mathbf{q}_{e}^{\prime}$ becomes unstable. This transition is precisely a Hopf bifurcation where the eigenvalues of the linearization about $\mathbf{q}_{e}^{\prime}$ cross the

\footnotetext{
${ }^{4}$ In case where the $\mathcal{P}$ is considered as a map from and to the original domain $\mathbb{R}^{n}$, its Jacobian matrix has an additional trivial eigenvalue that equals 1 , associated with initial perturbations which are tangent to the periodic solution, i.e., normal to $\mathcal{S}$.
}

Copyright $\odot$ by SIAM. Unauthorized reproduction of this article is prohibited. 


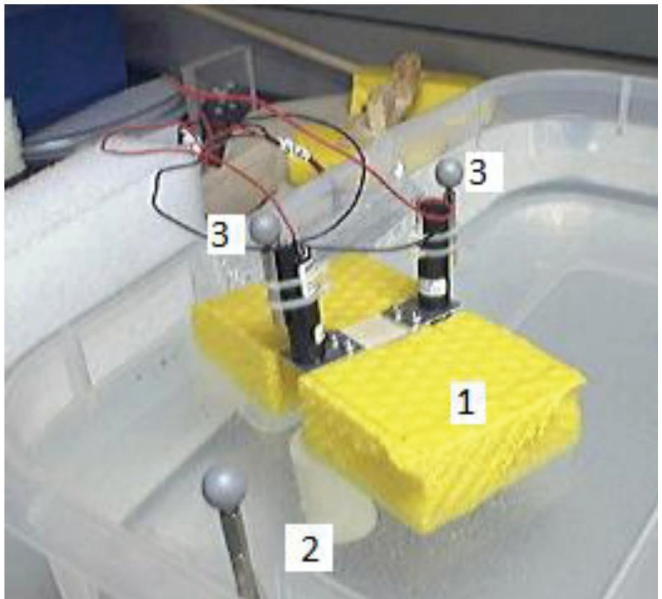

(a)

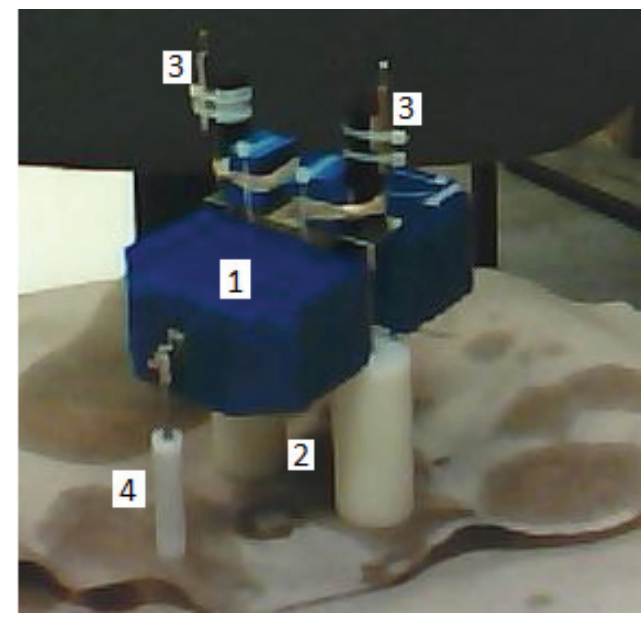

(b)

Figure 10. Pictures of (a) the 2-cylinder swimmer, and (b) the 2+1-cylinder swimmer. 1. foam flotation, 2. nylon cylinders (2.54 cm diameter), 3. motors/tracking markers, 4 . front cylinder (1 cm diameter).

imaginary axis, as shown in Figure 8(b). We could not find any periodic solutions for $l>7.22$, and numerical simulations resulted in rapid evolution of $\mathbf{q}^{\prime}(t)$ towards singular solutions of "crashing" into the wall. When $l$ decreases towards zero, the periodic solution becomes less and less stable $d \mathcal{P} / d y \rightarrow 1$. We could not find any periodic solutions for $0<l<0.5$, since they are very weakly stable and convergence becomes extremely slow. Unlike the case of $l=7.22$, the transition at $l=0$ is a degenerate Hopf bifurcation. This is due to the fact that for $l=0$ the system possesses a reversing symmetry which implies the existence of a continuum of marginally stable periodic orbits around $\mathbf{q}_{e}^{\prime}$ (the reversible Lyapunov center), as illustrated in the phase portrait of Figure 5(a). The range of periodic orbits at $l=0$ was determined numerically to be $y_{p} \in[0,104.25]$. Further investigation of this special bifurcation will be the subject of a future paper. Finally, for $l<0$ the picture is symmetric, where the stability characterization of solutions is simply reversed, as shown in Figure 9(c).

5. Experimental results. In this section we report the results of motion experiments on a macroscale robotic swimmer in a tank of viscous fluid. The experiments were carried out at the Laboratory of Autonomous Vehicles at the Department of Control and Dynamical Systems in Caltech. They are currently limited to verifying qualitative aspects of the theoretical predictions regarding steady translation parallel to a wall.

5.1. Description of the experimental setup. The first swimmer prototype consisted of two nylon cylinders rotated by DC motors. The cylinders were submerged in a container of viscous fluid, while the motors were mounted on a thin metal frame supported by a flotation cell made of foam. The goals of this flotation structure were to keep the DC motors outside of the fluid, support the weight of the robot, and confine its motion to a horizontal plane by suppressing all off-plane motions. A picture of the 2-cylinder swimmer appears in Figure 10(a). In order to keep the Reynolds number small in the macroscale system, the test fluid was chosen to be highly viscous silicone oil (Polydimethylsil-oxane), with kinematic viscosity of

Copyright (C) by SIAM. Unauthorized reproduction of this article is prohibited. 
$\nu=60,000 \mathrm{cSt}$ and specific gravity of 0.976 . The silicone oil was contained in a tank with dimensions of $110 \times 50 \times 17 \mathrm{~cm}$. The dimensions of the cylinders were $6.45 \mathrm{~cm}$ length and 2.54 $\mathrm{cm}$ diameter, and the spacing between their axes was $6.50 \mathrm{~cm}$. The reasons for using cylinders instead of the spheres considered in the theoretical model were the easier manufacturing and assembly and the propulsion enhancement due to larger surface area per given diameter. The cylinders were rotated by using Micromo 1319SR DC motors connected to a power supply with manually controlled input voltages. The angular velocity of each cylinder was approximately proportional to the motor input voltage. In air, the average angular velocity was 60 revolutions per minute (RPM) at input voltage of $3 \mathrm{~V}$. In silicone oil, the average angular velocity was $30 \mathrm{RPM}$ at $6 \mathrm{~V}$. Thus, the voltage-velocity ratio was reduced by a factor of four due to the resistance of the viscous fluid. The Reynolds number for this swimmer was calculated according to the formula $R e=\omega a d / \nu$, where $\omega$ is the angular velocity, $a$ is the radius of the cylinders, and $d$ is the center-to-center distance. The characteristic Reynolds number was found to be $R e=0.017$, which justifies the assumption of Stokes flow.

An infrared Optitrack Flex V:100 camera was used for tracking the motion of the swimmer. LEDs on the camera emit infrared light, and the lens picks up the reflection from reflective markers attached to the swimmer. Code was written using the Optitrack software development kit (SDK) in $\mathrm{C}++$ to track the markers positions. Suitable reflecting objects are determined and located in each frame through programmable threshold variables. These objects are then ranked based on programmable weighting variables. An extensive custom filtering algorithm was used to further suppress noises and guarantee tracking of the two desired markers. The output was calibrated against two markers mounted along the wall of the container serving as the reference for the $x$-axis. The sampling rate of the camera was $100 \mathrm{~Hz}$, but due to memory allocation limitations, we took one out of every five data points $(20 \mathrm{~Hz})$.

Two motion experiments were conducted with this swimmer near the wall, as follows. In the first experiment, the two cylinders were rotating at equal and opposite angular velocities under input voltages of $6 \mathrm{~V}$. In the second experiment, the input voltage to the motor closer to the wall was $5 \mathrm{~V}$, while the input voltage to the other motor was $6 \mathrm{~V}$, giving unequal angular velocities of the cylinders. Measurements from the optical tracking system were recorded for both runs. In order to demonstrate the theoretical predictions on the $2+1$-sphere swimmer, another swimmer prototype was constructed. This swimmer had further improvements for stability and rigidity with extra supports for the motors to maintain their vertical orientation. A third cylinder with diameter of $1 \mathrm{~cm}$ was attached rigidly to the front of the swimmer. A picture of this swimmer is shown in Figure 10(b). Experiments conducted with this swimmer include swimming near the wall with input voltages of $6 \mathrm{~V}$ and $5 \mathrm{~V}$ for which the cylinders are rotating at unequal angular velocities, under different initial distances from the wall.

5.2. Results. Position measurements from the tracking system were recorded during the motion experiments. The raw measurements from the tracking system were noisy. We identified a clear source of nearly periodic noise signal with frequency $0.5 \mathrm{~Hz}$, which is precisely the rotation frequency of the cylinders (30 RPM). We believe that the noise is a consequence of some small degree of eccentricity at the shafts and the cylinders caused by the set screws and additional misalignment, which excited weak lateral oscillations. In order to suppress these noises, all of the measured signals were filtered by a discrete-time low-pass Butterworth filter.

Copyright (c) by SIAM. Unauthorized reproduction of this article is prohibited. 


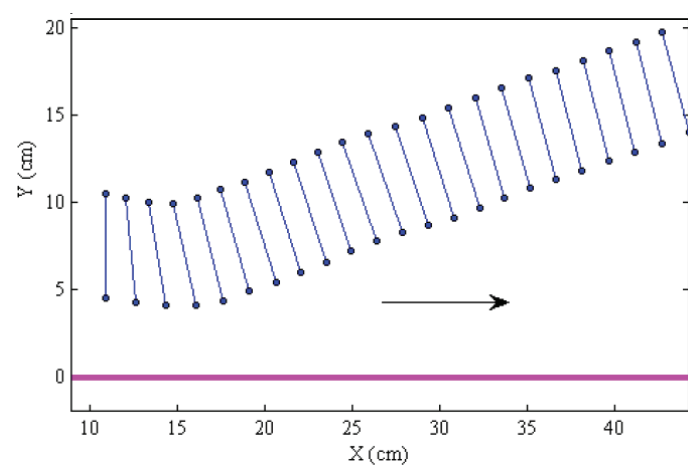

(a)

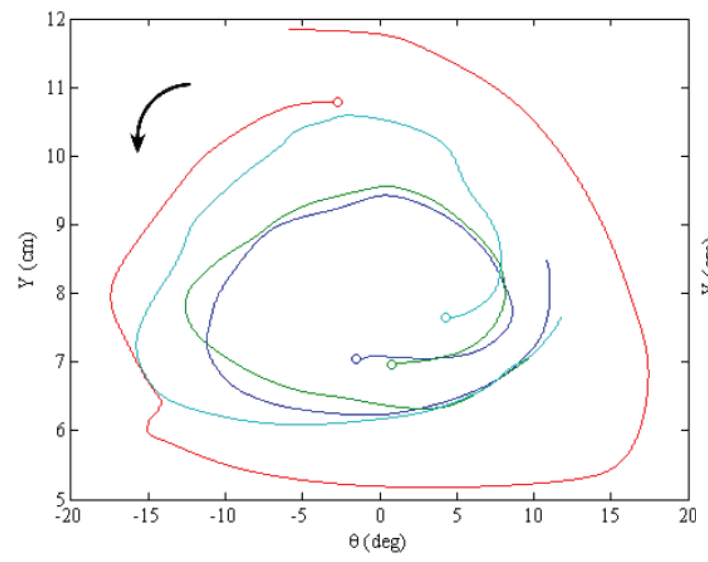

(c)

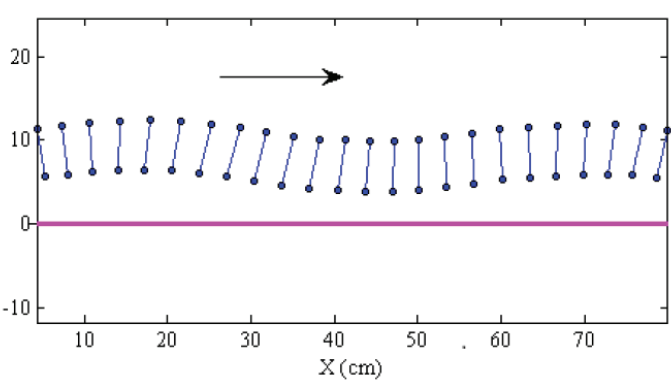

(b)

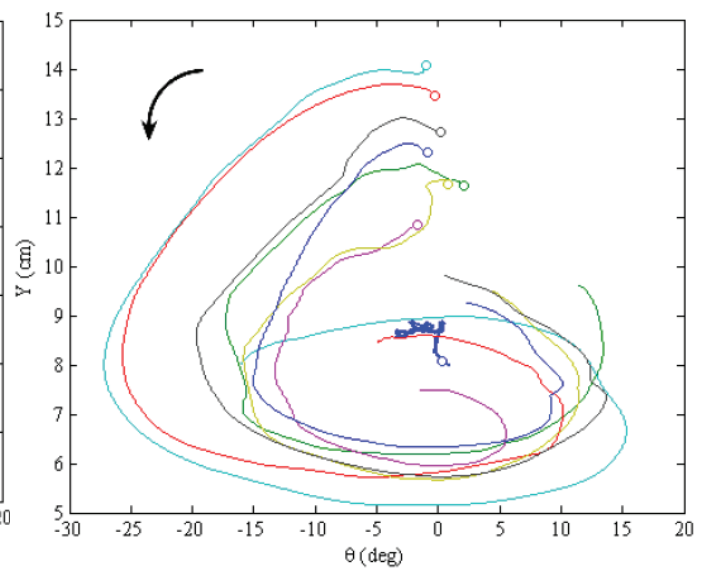

(d)

Figure 11. Experimental results. Motion snapshots of the 2-cylinder swimmer with (a) equal and (b) unequal motor angular velocities. Phase portraits of motion trajectories in the $(\theta, y)$-plane for (c) the 2-cylinder swimmer and (d) the $2+1$-cylinder swimmer with unequal motor angular velocities.

Video movies were taken by a web camera for representative runs and are available online at http://www.technion.ac.il/ izi/research/low_Re_swim/acc2010.

First, we recorded motion of the two-cylinder swimmer under equal and opposite angular velocities of the cylinders. When the swimmer was placed in the middle of the tank to simulate unbounded fluid, it moved roughly along a straight line with linear velocity of 1.5 $\mathrm{mm} / \mathrm{sec}$. When the swimmer was placed near the wall, it rotated and deviated away from the wall, as shown in the motion snapshots of Figure 11(a), which are taken from actual position measurements. The results agree with the theoretical prediction and the simulation shown in Figure 3(a). For the case of unequal input voltages, Figure 11(b) shows snapshots of the measured motion. The result is a nearly periodic motion, as predicted by the theory and shown in the simulated motion of Figure 6(a). Figure 11(c) shows phase portraits in the $(\theta, y)$-plane for the two-cylinder swimmer near the wall with unequal input voltages under four different initial conditions. Note that the trajectories are slightly diverging, in contrast to the theoretical prediction of perfectly closed orbits as in Figure 5(a). Possible reasons for this discrepancy are discussed in section 5.3. Figure 11(d) shows phase portraits in the $(\theta, y)$ plane for the $2+1$-cylinder swimmer near the wall under eight different initial conditions 
with unequal input voltages. The thick curve in the plot corresponds to a run where $y$ and $\theta$ are almost constant, indicating the existence of a stable equilibrium of fixed distance from the wall and fixed nonzero orientation. When an initial perturbation is imposed, the theory predicts asymptotic convergence towards the equilibrium point. Our experiments were conducted along a distance which was too short to demonstrate convergence. Nevertheless, using different initial conditions, the phase portrait in Figure 11(d) clearly shows a tendency of the trajectories to spiral in towards an equilibrium point, which indicates asymptotic stability, as predicted by the theory and shown in the simulated phase portrait in Figure 7(c).

5.3. Discussion. The results display qualitative agreement with the behavior predicted in the theory. We now list some discrepancies between the experimental results and the theoretical predictions and suggest possible explanations. First, note that trajectories of the two-cylinder swimmer with unequal angular velocities in Figure 11(c) are slightly diverging, in contrast to the theoretical prediction of perfectly closed orbits, shown in Figure 5(a). A possible explanation is that the exact periodic motion is a consequence of the swimmer being perfectly fore-aft symmetric in the horizontal plane. In the experimental system, misalignment of the flotation structure as well as small off-plane inclination of the swimmer during motion can add some degree of asymmetry which causes the slight divergence. Second, in some of the trajectories in the phase portrait of Figure 11(d) for the 2+1-cylinder swimmer, there is a noticeable transient in the beginning of each run until the trajectory is settled to a spiral curve. This transient can be explained by the duration until the DC power supply develops the desired input voltages once it is initially turned on. Third, in the phase portraits of Figures 11(c)11(d) for the 2-cylinder and 2+1-cylinder swimmers, some trajectories are intersecting each other, in contrast to the theoretical prediction shown in Figures 5(a) and 7(c), which is implied by uniqueness of solutions of the dynamical system. This can be explained by unmodelled effects that induce higher-order dynamics on top of the simple planar system considered in the theoretical model. Examples of such unmodelled effects are off-plane dynamics of the swimmer and inertial effects due to finite swimmer size. Other possible sources for discrepancies in the repeatability of runs are associated with the power supply and the DC motors. The assumption that setting input voltages is equivalent to setting velocities is not completely precise due to the unmodelled dynamics of the motor's electric circuit. Moreover, significant heating of the wires was observed during experiments, which may have changed the voltage-velocity ratio and affected the repeatability of the results. Another possible reason for deviation of the actual input voltages from their desired values is the low resolution of the manual voltage control in the DC power supply. Additionally, in several experimental runs, we observed events where one of the motors was stuck for a few seconds, possibly due to axis misalignment or internal shaft slippage, which could also explain some of the discrepancies. Finally, a major limitation of the experimental setup was the limited length of the tank relative to the size of the swimmer. This limitation precluded the possibility of measuring long-distance motion of the swimmers and observing steady-state convergence to either steady translation or periodic solutions. Possible future improvements of the experimental system are briefly discussed in the next section.

6. Conclusion. In this paper, we have analyzed the dynamics of a simple low-Re swimmer model near a wall. We have shown that fore-aft symmetry in the structure of the swimmer in-

Copyright (c) by SIAM. Unauthorized reproduction of this article is prohibited. 
duces a reversing symmetry of its dynamics, which, in turn, implies the existence of marginally stable periodic solutions of oscillating motion along the wall. When the fore-aft symmetry is broken by changing the swimmer's structure, the steady translation motion as well as the oscillating motion may become open-loop asymptotically stable, depending on the strength of symmetry breaking. Using numerical simulations, we have numerically characterized the dynamics and stability of solutions for the 2 -sphere and $2+1$-sphere swimmer models. The results were then verified qualitatively by conducting motion experiments with robotic low-Re swimmer prototypes.

We now briefly discuss limitations of the results and suggest possible directions for future extensions. We begin by discussing the theoretical part of this work. First, despite the relative simplicity of the swimmer model, its dynamic equations of motion are very complicated and do not admit closed-form expressions. The resulting dynamical system could be investigated only via numerical simulations, since it was analytically intractable. Thus, there is a need for a simpler model which will yield explicit dynamic formulation while still capturing the essential ingredients of the swimmer-wall hydrodynamic interactions. Such a model will be amenable to more detailed parametric analysis. A promising step in this direction has been obtained in the recent work [17], which considers a two-dimensional model of a symmetric circular treadmilling swimmer and utilizes methods of complex-variable analysis to find an effective singularity description of the system and derive simple closed-form expressions for the dynamics. Similar techniques have also been employed for more complex scenarios such as swimming beneath a deformable interface [16] and near a gap in the wall [18,62]. Another recent work [15] utilizes the Lorentz reciprocal theorem to derive the exact expressions for the dynamics of the two-dimensional treadmilling swimmer considered in [17]. It is believed that employing these techniques will result in explicit formulation of the swimming dynamics, which will be amenable to more thorough parametric study of the symmetry-breaking bifurcations discussed in section 4 . This analysis will hopefully give further physical insights into the behavior of more realistic three-dimensional swimmers near a boundary.

Second, in order to more reliably study biological swimmers such as bacteria and sperm cells, the model should be extended to account for a shape-changing swimmer near a wall. Two natural starting points are the one-dimensional three-linked-spheres swimmer model [53, 72] (Figure 1(a)) and Purcell's three-link swimmer [2, 3, 60] (Figure 1(c)), which displays the simplest form of flagellar-like traveling wave undulations. In both models, there are two degrees of freedom describing the swimmer's internal shape which are regarded as inputs, and net motion is generated by prescribing a cyclic change in the shape, called gait. As a preliminary result, a recent work which studies a chain-of-spheres version of Purcell's swimmer near a wall [54] analyzes the geometric symmetries in the dynamic equations and identifies a class of gaits that induces a reversing symmetry on the swimmer's discrete-time dynamics, which is analogous to the reversing symmetry studied here. Work in progress focuses on analysis of the dynamic stability and development of a more accurate numerical simulation scheme which will enable detailed parametric study.

Third, the dynamic model should be improved in order to account for additional physical effects in realistic biological scenarios, such as more geometrically complicated fluid domains, the presence of background shear flow, non-Newtonian (e.g., viscoelastic) fluid, leading-order effects of inertia, and so on. In order to improve the reliability and accuracy of simulations,

Copyright (c) by SIAM. Unauthorized reproduction of this article is prohibited. 


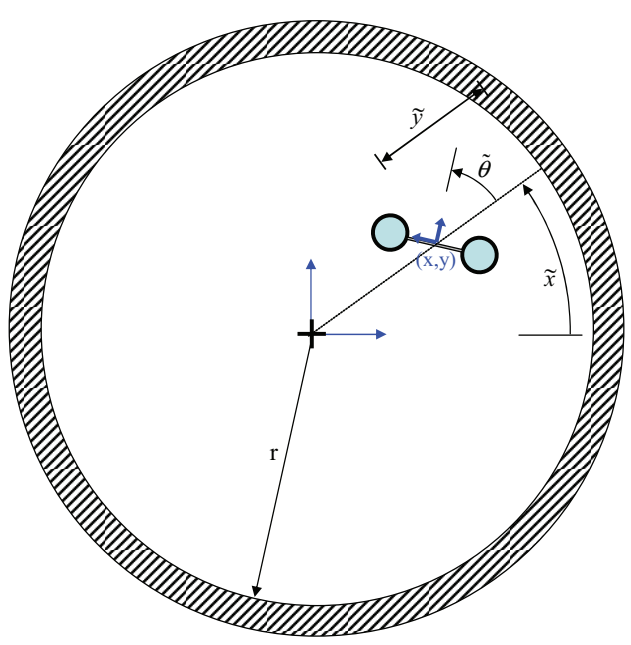

Figure 12. A swimmer in a circular fluid domain.

the problem should also be analyzed by using more powerful numerical techniques such as boundary element methods [59] and multipole expansions [24, 57] instead of the far-field approximations used here.

Another interesting theoretical model is motivated by future extension of the experimental setup, which is discussed in the remainder of this section. Consider a low-Re swimmer in a circular fluid domain, as shown in Figure 12. In this case, the fluid domain is completely bounded, and the infinite plane wall is replaced by the circular boundary of radius $r$. Fixing the origin at the center of the circle, the coordinates $\mathbf{q}=(x, y, \theta)$ describing the position and orientation of a frame attached to the swimmer's body can be replaced by the coordinates $\tilde{\mathbf{q}}=(\tilde{x}, \tilde{y}, \tilde{\theta})$, defined by

$$
\tilde{x}=\tan ^{-1}(y / x), \quad \tilde{y}=r-\sqrt{x^{2}+y^{2}}, \quad \tilde{\theta}=\theta-\tan ^{-1}(y / x) .
$$

That is, $\tilde{x}$ describes the motion along the boundary, $\tilde{y}$ describes the distance from the boundary (so that the boundary is again at $\tilde{y}=0$ ), and $\tilde{\theta}$ describes the swimmer's orientation with respect to the radius vector $(x, y)$; see Figure 12. Expressing the swimming equation $(20)$ with respect to the new coordinates $\tilde{\mathbf{q}}$, it is clear that $\mathbf{G}(\tilde{\mathbf{q}})$ is independent of the coordinate $\tilde{x}$. Moreover, considering the two-sphere swimmer (shown in Figure 2(b)) in a circular domain, its equation of motion satisfies exactly the same reversing symmetry in (23) with respect to the new coordinates $\tilde{\mathbf{q}}$. Therefore, Theorems 3.1 and 3.2 and the rest of the results of this paper will generalize directly to this case. Moreover, in case where the circle's radius $r$ is much greater than the swimmer's size, it is expected that the its dynamic behavior will be very similar to the case of an infinite plane wall.

Finally, in regard to our experimental results, the preliminary laboratory setup described here had two main limitations, as follows. First, the limited dimensions of the tank relative to the swimmer's size were not suitable for long-distance motion that demonstrates convergence to steady-state behavior. Second, the control of input voltages instead of angular velocities caused discrepancy between the theoretical model and the experiment, and perhaps even 
affected the repeatability of runs due to heating of the wires and inaccuracies in manually setting the voltages. Consequently, the experimental results reported here could corroborate the theoretical predictions only qualitatively. In an ongoing work at the Laboratory of BioDynamics and Mechanics of Locomotion at the Technion, we are working on designing an improved setup with a smaller swimmer that has direct control of the motors' angular velocities through magnetic encoders and a feedback control loop. Additionally, we have constructed a circular fluid tank and plan to conduct motion experiments in it as well. These future experiments will enable measurement of long-distance motion along the boundary, where we expect to observe complete convergence of the asymmetric swimmer to steady-state motion along the wall. Moreover, we plan to experimentally investigate the influence of system parameters on the stability characterization of periodic motion in order to complete the verification of theoretical findings of this paper.

\section{REFERENCES}

[1] J. E. Avron, O. Kenneth, And D. H. OAkmin, Pushmepullyou: An efficient micro-swimmer, New J. Phys., 7 (2005), pp. 234-238.

[2] J. E. Avron And O. RAz, A geometric theory of swimming: Purcell's swimmer and its symmetrized cousin, New J. Phys., 10 (2008), 063016.

[3] L. E. Becker, S. A. Koehler, And H. A. Stone, On self-propulsion of micro-machines at low Reynolds numbers: Purcell's three-link swimmer, J. Fluid Mech., 490 (2003), pp. 15-35.

[4] B. Behkam And M. Sitti, Design methodology for biomimetic propulsion of miniature swimming robots, ASME J. Dyn. Syst. Measurement Control, 128 (2006), pp. 36-43.

[5] A. P. Berke, L. Turner, H. C. Berg, and E. Lauga, Hydrodynamic attraction of swimming microorganisms by surfaces, Phys Rev. Lett., 101 (2008), 038102.

[6] J. R. Blake, Self propulsion due to oscillations on the surface of a cylinder at low Reynolds number, Bull. Austral. Math. Soc., 5 (1971), pp. 255-264.

[7] J. R. Blake, A spherical envelope approach to ciliary propulsion, J. Fluid Mech., 46 (1971), pp. 199-208.

[8] J. R. Blake And A. T. Chwang, Fundamental singularities of viscous-flow. Part 1. Image systems in vicinity of a stationary no-slip boundary, J. Engrg. Math., 8 (1974), pp. 23-29.

[9] H. Brenner, Effect of finite boundaries on the Stokes resistance of an arbitrary particle, J. Fluid Mech., 12 (1962), pp. 35-48.

[10] F. Bullo and A. D. Lewis, Geometric Control of Mechanical Systems, Springer, New York, 2005.

[11] R. E. Caflisch, C. Lim, J. H. C. Luke, and A. S. Sangani, Periodic solutions for three sedimenting spheres, Phys. Fluids, 31 (1988), pp. 3175-3179.

[12] S. Childress, Mechanics of Swimming and Flying, Cambridge University Press, Cambridge, UK, 1981.

[13] J. ConTÉs, Geometric, Control, and Numerical Aspects of Nonholonomic Systems, Springer-Verlag, Berlin, Heidelberg, 2002.

[14] J. Cosson, P. Huitorel, And C. Gagnon, How spermatozoa come to be confined to surfaces, Cell Motility Cytoskeleton, 54 (2003), pp. 56-63.

[15] D. G. CRowdy, Treadmilling swimmers near a no-slip wall at low Reynolds number, Internat. J. NonLinear Mech., 46 (2011), pp. 577-585.

[16] D. G. Crowdy, S. Lee, O. Samson, A. E. Hosoi, and E. Lauga, A two dimensional model of low Reynolds number swimmers beneath a free surface, J. Fluid Mech., 681 (2011), pp. 24-47.

[17] D. G. CRowdy And Y. OR, Two-dimensional point singularity model of a low Reynolds number swimmer near a wall, Phys. Rev. E, 81 (2010), 036313.

[18] D. G. Crowdy And O. SAmson, Hydrodynamic bound states of a low-Reynolds-number swimmer near a gap in a wall, J. Fluid Mech., 667 (2011), pp. 309-335.

[19] R. L. Devaney, Reversible diffeomorphisms and flows, Trans. Amer. Math. Soc., 218 (1976), pp. 89-113.

[20] K. Drescher, K. C. Leptos, I. Tuval, T. Ishikawa, T. J. Pedley, and R. E. Goldstein, Dancing Volvox: Hydrodynamic bound states of swimming algae, Phys. Rev. Lett., 102 (2009), 168101.

Copyright $\odot$ by SIAM. Unauthorized reproduction of this article is prohibited. 
[21] R. Dreyfus, J. Baudry, M. L. Roper, M. Fermigier, H. A. Stone, and J. Bibette, Microscopic artificial swimmers, Nature, 437 (2005), pp. 862-865.

[22] L. J. Durlofsky, J. F. Brady, And G. Bossis, Dynamic simulation of hydrodynamically interacting particles, J. Fluid Mech., 180 (1987), pp. 21-49.

[23] L. J. Faudi And A. McDonald, Sperm motility in the presence of boundaries, J. Bull. Math. Biol., 57 (1995), pp. 679-699.

[24] A. V. Filippov, Drag and torque on clusters of $N$ arbitrary spheres at low Reynolds number, J. Colloid Interface Sci., 229 (2000), pp. 184-195.

[25] P. D. Frymier, R. M. Ford, H. C. Berg, and P. T. Cummings, Three-dimensional tracking of motile bacteria near a solid planar surface, Proc. Natl. Acad. Sci. USA, 92 (1995), pp. 6195-6199.

[26] A. Ghosh And P. Fischer, Controlled propulsion of artificial magnetic nanostructured propellers, Nano Letters, 9 (2009), pp. 2243-2245.

[27] M. Golubitsky, M. Krupa, And C. Lim, Time-reversibility and particle sedimentation, SIAM J. Appl. Math., 51 (1991), pp. 49-72.

[28] T. Goto, K. Nakata, K. Baba, M. Nishimura, and Y. Magariyama, A fluid-dynamic interpretation of the asymmetric motion of singly flagellated bacteria swimming close to a boundary, Biophys. J., 89 (2005), pp. 3771-3779.

[29] J. Guckenheimer And P. Holmes, Nonlinear Oscillations, Dynamical Systems, and Bifurcations of Vector Fields, Springer-Verlag, New York, 1983.

[30] J. Happel And H. Brenner, Low Reynolds Number Hydrodynamics, Prentice-Hall, Englewood Cliffs, NJ, 1965.

[31] P. Hartman, A lemma in the theory of structural stability of differential equations, Proc. Amer. Math. Soc., 11 (1960), pp. 610-620.

[32] T. Ishikawa, M. P. Simmonds, And T. J. Pedley, Hydrodynamic interaction of two swimming model micro-organisms, J. Fluid Mech., 568 (2006), pp. 119-160.

[33] G. B. Jeffrey, The motion of ellipsoidal particles immersed in a viscous fluid, Proc. R. Soc. London, 102 (1922), pp. 161-169.

[34] S. Jung, S. E. Spagnolie, K. Parikh, M. Shelley, and A.-K. Tornberg, Periodic sedimentation in a Stokesian fluid, Phys. Rev. E, 74 (2006), 035302(R).

[35] E. Kanso, J. E. Marsden, C. W. Rowley, and J. B. Melli-Huber, Locomotion of articulated bodies in a perfect fluid, J. Nonlinear Sci., 15 (2005), pp. 255-289.

[36] D. F. KATZ, On the propulsion of micro-organisms near solid boundaries, J. Fluid Mech., 64 (1974), pp. 33-49.

[37] D. F. Katz, J. R. Blake, and S. L. Paverifontana, Movement of slender bodies near plane boundaries at low Reynolds number, J. Fluid Mech., 72 (1975), pp. 529-540.

[38] T. Kaya And H. Koser, Characterization of hydrodynamic surface interactions of Escherichia Coli cell bodies in shear flow, Phys Rev. Lett., 103 (2009), 138103.

[39] S. D. Kelly and R. M. Murray, Geometric phases and robotic locomotion, J. Robotic Systems, 12 (1995), pp. 417-431.

[40] S. Kim and S. J. Karrila, Microhydrodynamics, Butterworth-Heinemann, Boston, 1991.

[41] J. Koiller, R. Montgomery, And K. Ehlers, Problems and progress in microswimming, J. Nonlinear Sci., 6 (1996), pp. 507-541.

[42] G. Kosa, M. Shoham, and M. ZaAroor, Propulsion method for swimming micro robots, IEEE Trans. Robotics, 23 (2007), pp. 137-150.

[43] J. S. W. Lamb, Reversing symmetries in dynamical systems, J. Phys. A, 25 (1992), pp. 925-937.

[44] J. S. W. Lamb And J. A. G. Roberts, Time-reversal symmetry in dynamical systems: A survey, Phys. D, 112 (1998), pp. 1-39.

[45] E. Lauga, W. R. Diluzio, G. M. Whitesides, and H. A. Stone, Swimming in circles: Motion of bacteria near solid boundaries, Biophys. J., 90 (2006), pp. 400-412.

[46] E. Lauga And T. R. Powers, The hydrodynamics of swimming microorganisms, Rep. Progr. Phys., 72 (2009), 096601.

[47] A. M. Leshansky and O. Kenneth, Surface tank treading: Propulsion of Purcell's toroidal swimmer, Phys. Fluids, 20 (2008), 063104.

Copyright (C) by SIAM. Unauthorized reproduction of this article is prohibited. 
[48] A. M. Leshansky, O. Kenneth, O. Gat, and J. E. Avron, A frictionless microswimmer, New J. Phys., 9 (2007), pp. 145-161.

[49] M. J. LighthiLl, On the squirming motion of nearly spherical deformable bodies through liquids at very small Reynolds numbers, Comm. Pure Appl. Math., 5 (1952), pp. 109-118.

[50] J. E. Marsden, Lectures on Mechanics, Cambridge University Press, New York, 1992.

[51] J. Melli And C. W. Rowley, Models and control of fish-like locomotion, Exper. Mech., 50 (2010), pp. $1355-1360$.

[52] J. B. Melli, C. W. Rowley, And D. S. Rufat, Motion planning for an articulated body in a perfect planar fluid, SIAM J. Appl. Dyn. Syst., 5 (2006), pp. 650-669.

[53] A. Najafi And R. Golestanian, Simple swimmer at low Reynolds numbers: Three linked spheres, Phys. Rev. E, 69 (2004), pp. 062901-062904.

[54] Y. OR, Dynamics and stability of Purcell's three-link microswimmer near a wall, Phys. Rev. E, 82 (2010), $065302(\mathrm{R})$

[55] Y. Or And R. M. Murray, Dynamics and stability of a class of low Reynolds number swimmers near a wall, Phys. Rev. E, 79 (2009), 045302(R).

[56] J. Ostrowski And J. BuRdick, The geometric mechanics of undulatory robotic locomotion, Internat. J. Robot. Res., 17 (1998), pp. 683-701.

[57] S. S. OzARkAR AND A. S. SANGAni, A method for determining Stokes flow around particles near a wall or in a thin film bounded by a wall and a gas-liquid interface, Phys. Fluids, 20 (2008), 063301.

[58] T. J. Pedley and J. O. Kessler, Hydrodynamic phenomena in suspensions of swimming microorganisms, Annu. Rev. Fluid Mech., 24 (1992), pp. 313-358.

[59] C. PozRIKIdis, Boundary Integral and Singularity Methods for Linearized Viscous Flow, Cambridge University Press, Cambridge, UK, 1992.

[60] E. M. Purcell, Life at low Reynolds number, Amer. J. Phys., 45 (1977), pp. 3-11.

[61] M. Ramia, D. L. Tullock, And N. Phan-Thien, The role of hydrodynamic interaction in the locomotion of microorganisms, Biophys. J., 65 (1993), pp. 755-778.

[62] O. SAmson, Low Reynolds Number Swimming in Complex Environments, Ph.D. thesis, Department of Mathematics, Imperial College, London, UK, 2010; available online at http://www2.imperial.ac.uk/ $\sim$ dgcrowdy/_students/SamsonThesis.pdf.

[63] M. B. Sevryuk, Reversible Systems, Lecture Notes in Math. 1211, Springer-Verlag, Berlin, 1986.

[64] E. A. Shammas, H. Choset, And A. A. Rizzi, Geometric motion planning analysis for two classes of underactuated mechanical systems, Internat. J. Robot. Res., 26 (2007), pp. 1043-1073.

[65] A. Shapere And F. WilczeK, Geometry of self-propulsion at low Reynolds numbers, J. Fluid Mech., 198 (1989), pp. 557-585.

[66] H. Shum, E. A. Gaffney, And D. J. Smith, Modelling bacterial behaviour close to a no-slip plane boundary: The influence of bacterial geometry, Proc. Roy. Soc. A, 466 (2010), pp. 1725-1748.

[67] D. J. Sмith and J. R. Blake, Surface accumulation of spermatozoa: A fluid dynamic phenomenon, Math. Sci., 34 (2009), pp. 74-87.

[68] J. W. SWAN AND J. F. BRAdy, Simulation of hydrodynamically interacting particles near a no-slip boundary, Phys. Fluids, 19 (2007), 113306.

[69] G. I. TAYLOR, Analysis of the swimming of microscopic organisms, Proc. Roy. Soc. A, 209 (1951), pp. 447461.

[70] P. Tierno, R. Golestanian, I. Pagonabarraga, and F. Sagues, Controlled swimming in confined fluids of magnetically actuated colloidal rotors, Phys. Rev. Lett., 101 (2008), 218304.

[71] E. YARIV, Self-propulsion in a viscous fluid: Arbitrary surface deformations, J. Fluid Mech., 550 (2006), pp. 139-148.

[72] R. Zargar, A. NAJAfi, And M. MiRI, Three-sphere low-Reynolds-number swimmer near a wall, Phys. Rev. E, 80 (2009), 026308.

[73] S. Zhang, Y. OR, And R. M. Murray, Experimental demonstration of the dynamics and stability of a low Reynolds number swimmer near a plane wall, in Proceedings of the American Control Conference, Baltimore, MD, IEEE Press, Piscataway, NJ, 2010, pp. 4205-4210.

[74] G. Zilman, J. Novak, and Y. Benayahu, How do larvae attach to a solid in a laminar flow?, Mar. Biol., 154 (2008), pp. 1-26.

Copyright (C) by SIAM. Unauthorized reproduction of this article is prohibited. 